\title{
Flexible Electrodes for Supercapacitors
}

\section{Based on the Supramolecular Assembly of}

\section{Biohydrogel and Conducting Polymer}

\section{Mari Cruz G. Saborío, ${ }^{1,2}$ Sonia Lanzalaco, ${ }^{1,2}$ Georgina Fabregat, ${ }^{1,2}$ Jordi Puiggalí, ${ }^{1,2}$ Francesc Estrany, ${ }^{1,2, *}$ and Carlos Alemán ${ }^{1,2, *}$}

${ }^{1}$ Departament d'Enginyeria Química, EEBE, Universitat Politècnica de Catalunya, C/ Eduard Maristany, 10-14, Ed. I2, 08019, Barcelona, Spain

${ }^{2}$ Barcelona Research Center for Multiscale Science and Engineering, Universitat Politècnica de Catalunya, Eduard Maristany, 10-14, 08019, Barcelona, Spain

\footnotetext{
*Corresponding authors: francesc.estrany@upc.edu and carlos.aleman@upc.edu
} 


\begin{abstract}
Flexible and lightweight electrodes are prepared using a two-step process. First, poly(3,4ethylenedioxythiophene) (PEDOT) microparticles are loaded into poly- $\gamma$-glutamic acid ( $\gamma$-PGA) hydrogel matrix, during the reaction of the biopolymer chains with the cross-linker, cystamine. After this, PEDOT particles dispersed inside the hydrogel are used as polymerization nuclei for the chronoamperometric synthesis of poly(hydroxymethyl-3,4-ethylenedioxythiophene) (PHMeDOT) in aqueous solution. After characterization of the resulting electrode composites, electrochemical studies revealed that the capacitive properties drastically depend on the polymerization time used to produce PHMeDOT inside the loaded hydrogel matrix. Specifically, flexible electrodes obtained using a polymerization time of 7 hours exhibit an specific capacitance of $45.4 \pm 0.7 \mathrm{mF} / \mathrm{cm}^{2}$ from cyclic voltammetry and charge-discharge long-term stability. The applicability of these electrodes in lightweight and flexible energy-harvesting systems useful for energy-autonomous, low-power, disposable electronic devices, has been proved powering a LED bulb.
\end{abstract}




\section{Introduction}

A great interest in thin, flexible, safe energy storage devices has been shown by the scientific community over the last decades. ${ }^{1,2}$ Fully pliable and robust devices, conceivably and preferably composed of eco-friendly materials, are the new benchmark of modern society. ${ }^{1}$ These devices have a large variety of applications from motor vehicles ${ }^{3-5}$ to laptops ${ }^{6,7}$ or autonomous medical sensors. ${ }^{8,9}$ Energy can be stored in batteries or in capacitors; the main difference is the charge storage mechanism, which is based on faradic and non-faradic processes, respectively. In the former devices, an electron transfer that produces a redox reaction takes place, whereas the second type is based on electrostatic processes that occur in absence of electron transfer across the electrode interface. ${ }^{10}$ Conventional capacitors share important similarities with another class of devices known as electrochemical capacitors, which rely on charge separation at electrode/electrolyte interfaces to store energy.

Electrochemical capacitors have superb specific power compared to batteries, but modest specific energy. Batteries are characterized by high energy density values of $10-100 \mathrm{Wh} / \mathrm{kg}$, whereas capacitors are able to release the stored energy much faster however the energy density is $<0.1 \mathrm{Wh} / \mathrm{kg} .{ }^{11}$ Recently, electrochemical supercapacitors (ESCs) have emerged displaying higher energy values (i.e. $1-10 \mathrm{Wh} / \mathrm{kg}$ ) compared to capacitors. ${ }^{12}$ ESCs present an interface between an electronic conductor and an ionic conductor (i.e. the electrolyte).$^{13}$ The simplest ESC is composed of two non-reactive porous electrodes immersed into an electrolytic medium and electrically isolated by a membrane to allow the migration of ions. From a technological point of view, ESCs are characterized by a good acceleration, robustness and excellent life cycle, which 
can improve the effectiveness of battery-based systems by shrinking the volume of the batteries and reducing the frequency of their replacement. ${ }^{10}$

In spite of the advantages reported for ESCs, the quality of these devices has to be improved by using more environmentally friendly materials (e.g. renewable materials) and electrolytes (e.g. non-organic, aqueous solvents), and by improving properties such as capacitance, flexibility and durability. These improvements could be obtained through:

- Construction of 3D devices incorporating micro- and/or nanometric conductive components arranged in interpenetrating networks, with the aim to create short diffusional paths and, thus, very high currents.

- Replacement of organic solvents by water-containing gel-biopolymer electrolytes, characterized by ease of processability, large exposed area for electrochemical activity, good resistance to strain and, in addition, significant reduction of costs.

Within this context, we have developed the supra-molecular assembly of a biohydrogel with a conducting polymer $(\mathrm{CP})$, producing and characterizing a new flexible, lightweight and efficient organic electrode for application in ESCs. More specifically, we have prepared an electrode composed by poly- $\gamma$-glutamic acid $(\gamma$-PGA) as 3D-gelated network and a poly $(3,4-$ ethylenedioxythiophene) (PEDOT) derivative, as CP.

Selected polymers are characterized by high relevance in research and industrial environments. $\gamma$-PGA is an anionic homopolypeptide linked by the peptide bond between the $\alpha$ amino and the $\gamma$-carboxyl groups of glutamic acid, ${ }^{14}$ that exhibits good biocompatibility due to its biodegradability, water-solubility and non-toxicity towards humans. ${ }^{15}$ This compound is naturally synthetized as a slime layer by a variety of members of the genus Bacillus. ${ }^{16} \gamma$-PGA and its 
derivatives have been used in different fields such as food industry, ${ }^{17,18}$ medicine, ${ }^{19,20}$ cosmetic, ${ }^{21,22}$ agriculture, ${ }^{23}$ and wastewater treatment. ${ }^{24}$ Due to their robustness, $\gamma$-PGA gels were recently employed as solid electrolyte media for organic ESCs. ${ }^{25}$ Armelin et $a .^{26}$ recently reviewed the utilization and the advantages of biohydrogels for ESCs, highlighting the sustainability of devices composed by materials that can be naturally produced, as $\gamma$-PGA from biosynthesis, or directly extracted from biomass.

Among CPs, PEDOT is one of the most widely used for energy storage devices due to its excellent properties: low band gap, easiness to stabilize the oxidized state, high electrical conductivity, stable charge-discharge response, excellent environmental stability and fast dopingdedoping process. ${ }^{27-30}$ The unique characteristics of PEDOT are due to the oxygen atoms attached at the $\beta, \beta^{\prime}$-positions of the thiophene ring, which induce strong electron-donating effects and prevent the formation of parasitic $\alpha-\beta$ linkages during the polymerization of the 3,4ethylenedioxythiophene (EDOT) monomers. ${ }^{31}$ Moreover, the electrochemical performance of PEDOT can be improved if properly combined with other materials such as graphene, ${ }^{23,32-34}$ carbon nanotubes ${ }^{35}$ inorganic oxides, ${ }^{36,37}$ and even biomolecules. ${ }^{26,38-41}$

The present work represents a step ahead with respect to the setup of ESCs composed of PEDOT electrodes and $\gamma$-PGA solid electrolytic medium. More specifically, $\gamma$-PGA biohydrogel has been synthesized and analyzed in presence of PEDOT and poly(hydroxymethyl-3,4ethylenedioxythiophene) (PHMeDOT), a PEDOT derivative with an exocyclic hydroxyl group that facilitates its preparation in aqueous environments. Accordingly, the novelty of this work is related with the loading of PEDOT particles into the hydrogel, which are subsequently used as nucleation sites for the in situ electropolymerization of PHMeDOT inside the hydrated $\gamma$-PGA 
matrix. The excellent properties of the resulting electrode composite, the contribution of its different components (i.e. $\gamma$-PGA, PEDOT particles and PHMeDOT), and its potential applicability are discussed in the next sections.

\section{Materials and methods}

\section{Materials}

Free-acid $\gamma$-PGA (from Bacillus subtilis), with average molecular weight $\mathrm{M}_{\mathrm{w}}=350000$, was purchased from Wako Chemicals GmbH (Neuss, Germany). Cystamine dihydrochloride ( $\geq 98.0 \%$ ), 1-[3-(dimethylamino)propyl]-3-ethylcarbodiimide methiodide (EDC), EDOT (95\%) and hydroxymethyl-3,4-ethylenedioxythiophene (HMeDOT, 95\%) were purchased from SigmaAldrich. Acetonitrile (Reagent European Pharmacopoeia for analysis, ACS) and $\mathrm{NaHCO}_{3}$ were obtained from Panreac. Anhydrous lithium perchlorate $\left(\mathrm{LiClO}_{4}\right)$, analytical reagent grade from Aldrich, was stored in an oven at $70{ }^{\circ} \mathrm{C}$ before use in electrochemical experiments. Milli-Q water grade $(0.055 \mathrm{~S} / \mathrm{cm})$ was used in all synthetic processes.

\section{Synthesis of PEDOT particles}

Both anodic polymerization and electrochemical assays were performed with a potentiostatgalvanostat Autolab PGSTAT101 equipped with the ECD module (Ecochimie, The Netherlands) using a three-electrode compartment cell under nitrogen atmosphere $(99.995 \%$ pure) at room temperature. Steel AISI 316 sheets of $6 \mathrm{~cm}^{2}$ in area were used as working and counter electrodes, respectively. To prevent interferences during the electrochemical assays, the working and counter

electrodes were cleaned with acetone, ethanol and distillated water before each trial. The 
reference electrode was an $\mathrm{Ag} \mid \mathrm{AgCl}$ electrode containing a $\mathrm{KCl}$ saturated aqueous solution (offset potential versus the standard hydrogen electrode, $E^{0}=0.222 \mathrm{~V}$ at $25^{\circ} \mathrm{C}$ ).

PEDOT films were prepared by chronoamperometry (CA) applying a constant potential of $1.40 \mathrm{~V}$ during $600 \mathrm{~s}$ and using a $10 \mathrm{mM}$ monomer solution in acetonitrile with $0.1 \mathrm{M} \mathrm{LiClO}_{4}$ as reaction medium. The resulting films were processed into particles by sonication (Bandelin Sonopuls sonicator) in $0.5 \mathrm{M} \mathrm{NaHCO}_{3}$ solution during 25 min in steps of 5 min at low frequency ( $0.01 \%$ frequency). The diameter of the resulting PEDOT particles was determined at room temperature by dynamic light scattering (DLS) in Milli-Q water dispersions $(0.3 \% \mathrm{v} / \mathrm{v})$ using a NanoBrook Omni Zeta Potential Analyzer from Brookheaven Instruments Corporation. Finally, the basic aqueous $\left(0.5 \mathrm{M} \mathrm{NaHCO}_{3}\right)$ dispersion with $20 \% \mathrm{w} / \mathrm{w}$ of the resulting PEDOT particles was directly used for the synthesis of CP-loaded $\gamma$-PGA hydrogels (see below).

\section{Synthesis of the $\gamma-P G A$ hydrogel}

$\gamma$-PGA hydrogels were prepared adapting the procedure described by Matsusaki et $a .^{42} \gamma$ PGA and EDC were dissolved in $0.75 \mathrm{~mL}$ of $0.5 \mathrm{M} \mathrm{NaHCO}_{3}$ at $4{ }^{\circ} \mathrm{C}$ under magnetic stirring. Then, cystamine dihydrochloride, previously dissolved in $0.25 \mathrm{~mL}$ sodium hydrogen carbonate solution $(0.5 \mathrm{M})$, was added to the solution and mixed during 2-3 minutes. The $\gamma$-PGA / EDC / cystamine molar ratio was 5 / 5 / 4. The final solution was removed with a magnetic stirrer, and the reaction solution was poured into glass molds of $2.5 \times 1.5 \times 0.1 \mathrm{~cm}$. The solution was let to gel at room temperature for one hour. To remove any compound in excess, the resulting hydrogel was washed with distillate water three times. 


\section{CP-loaded $\gamma$-PGA hydrogels: PEDOT/ $\gamma$-PGA}

The procedure described in Section 2.3 was also used to prepare $\gamma$-PGA hydrogels loaded with PEDOT particles, hereafter denoted PEDOT/ $\gamma$-PGA. More specifically, the only difference with respect to the preparation of pure $\gamma$-PGA hydrogel is that the $0.5 \mathrm{M} \mathrm{NaHCO}_{3}$ solution used to dissolve the biopolymer already contained the PEDOT particles (20\% w/w of PEDOT particles with respect to the weight of $\gamma$-PGA).

Preparation of [PEDOT/ $\gamma-P G A] P H M e D O T$ electrodes through polymerization inside $P E D O T / \gamma-P G A$

Steel AISI 316 sheets of $0.5 \times 0.5 \mathrm{~cm}^{2}$ were coated with PEDOT/ $\gamma$-PGA hydrogel and subsequently kept into the reaction medium overnight whilst stirring $(65 \mathrm{rpm})$. The PEDOT/ $\gamma$ PGA coated sheets were then used as working electrodes for the anodic polymerization of PHMeDOT by CA. The reaction medium was a $10 \mathrm{mM}$ HMeDOT aqueous solution with $0.1 \mathrm{M}$ $\mathrm{LiClO}_{4}$ as supporting electrolyte. The anodic polymerization was conducted under a constant potential of $1.10 \mathrm{~V}$ using a polymerization time, $\theta$, of $6 \min$ or 7 hours. Thus, in a preliminary study (Figure S1) we observed that such two values are representative of systems obtained using $\theta$ lower and higher than $\sim 2 \mathrm{~h}$, respectively. The experimental setup used for the in situ modification of the PEDOT/ $\gamma$-PGA hydrogel was identical to that described in Section 2.2 for the synthesis of PEDOT films. Hereafter, the loaded PEDOT $/ \gamma$-PGA hydrogel electrochemically modified with PHMeDOT is denoted [PEDOT/ $\gamma$-PGA]PHMeDOT

\section{Morphological and topographical characterization}


Scanning electron microscopy (SEM) studies were performed to examine the surface morphology of PEDOT, PEDOT/ $\gamma-\mathrm{PGA}$ and $[\mathrm{PEDOT} / \gamma-\mathrm{PGA}] \mathrm{PHMeDOT}$ electrodes. Dried samples were placed in a Focussed Ion Beam Zeis Neon 40 scanning electron microscope operating at $3 \mathrm{kV}$, equipped with an energy dispersive X-ray (EDX) spectroscopy system. EDX analyses were performed to identify the presence of PEDOT particles and the success of the in situ PHMeDOT polymerization.

Atomic force microscopy (AFM) images were obtained with a Molecular Imaging PicoSPM using a NanoScope IV controller under ambient conditions. The tapping mode AFM was operated at constant deflection. The row scanning frequency was set to $1 \mathrm{~Hz}$. AFM measurements were performed on various parts of the films, which provided reproducible images similar to those displayed in this work. The scan window sizes used in this work were $5 \times 5 \mu \mathrm{m}^{2}$. The statistical application of the NanoScope Analysis software was used to determine the root mean square roughness $\left(\mathrm{R}_{\mathrm{q}}\right)$, which is the average height deviation taken from the mean data plane.

\section{Electrochemical characterization.}

All electrochemical experiments were run in triplicate using water with $0.1 \mathrm{M} \mathrm{LiClO}_{4}$ as supporting electrolyte. Cyclic voltammetry (CV) was carried out to evaluate the electroactivity, areal specific capacitance $(S C)$ and the electrochemical stability of the prepared electrodes. The initial and final potentials were $-0.50 \mathrm{~V}$, and the reversal potential was $1.10 \mathrm{~V}$. A scan rate of 100 $\mathrm{mV} / \mathrm{s}$ was used in all cases.

The areal $S C$ (in $\mathrm{mF} / \mathrm{cm}^{2}$ ) was determined using the following expression:

$$
S C=\frac{Q}{\Delta V \cdot A}
$$


where $Q$ is voltammetric charge determined by integrating the oxidative or the reductive parts of the cyclic voltammogram curve, $\Delta V$ is the potential window (in $\mathrm{V}$ ), and $A$ is the area of the electrode $\left(\right.$ in $\mathrm{cm}^{2}$ ). The exposed area of the different electrodes for $\mathrm{CV}$ analyses was $0.025 \mathrm{~cm}^{2}$. The electrochemical stability was examined by evaluating the loss of electroactivity (LEA, in \%) against the number of oxidation-reduction cycles:

$$
L E A=\frac{\Delta Q}{Q_{2}}=\frac{Q_{i}-Q_{2}}{Q_{2}}
$$

where $\Delta Q$ is the difference between the oxidation charge (in C) of the second $\left(Q_{2}\right)$ and the evaluated oxidation-reduction cycle $\left(\mathrm{Q}_{i}\right)$.

Electrochemical impedance spectroscopy (EIS) diagrams were taken at open circuit (OCP) over the frequency range of $100 \mathrm{kHz}$ to $10 \mathrm{mHz}$ with a potential amplitude of $0.05 \mathrm{~V}$ using an AUTOLAB-302N potentiostat/galvanostat. All experiments were performed at room temperature in water with $0.1 \mathrm{M} \mathrm{LiClO}_{4}$.

Galvanostatic charge-discharge (GCD) cycles were run between -0.50 and $0.40 \mathrm{~V}$ using a current of $0.1 \mathrm{~mA}$. GCD curves were also employed to evaluate the areal $S C$ according to:

$$
S C=\frac{\int I \cdot \Delta t}{\Delta V \cdot A}
$$

where $I$ is the applied current, $\Delta t$ is the time of discharge (in s), $\Delta V$ is the difference between the potential at the beginning and at the end of the discharge (in $\mathrm{V}$ ) and $A$ is the area of the electrode (in $\mathrm{cm}^{2}$ ).

Thermal stability, swelling and spectroscopic characterization 
The thermal stability of the prepared samples was studied by thermogravimetry (TGA) at a heating rate of $20^{\circ} \mathrm{C} / \mathrm{min}$ (sample weight $c a .5 \mathrm{mg}$ ) with a Q50 thermogravimetric analyzer of TA Instruments and under a flow of dry nitrogen. Test temperatures ranged from 30 to $600{ }^{\circ} \mathrm{C}$.

The swelling ratio $(S R, \%)$ of the hydrogels was determined according to:

$$
S R=\frac{w_{W}-w_{D}}{w_{D}}
$$

where $w_{W}$ is the weight of the hydrogels after $30 \mathrm{~min}$ in milli-Q water and $w_{D}$ is the weight of the hydrogel dried at room temperature during 30 min after preparation.

Samples were characterized by micro-Raman spectroscopy using a commercial Renishaw inVia Qontor confocal Raman microscope. The Raman setup consisted of a laser (at $785 \mathrm{~nm}$ with a nominal $300 \mathrm{~mW}$ output power) directed through a microscope (specially adapted Leica DM2700 M microscope) to the sample after which the scattered light is collected and directed to a spectrometer with a 1200 lines $\mathrm{mm}^{-1}$ grating. The exposure time was $10 \mathrm{~s}$, the laser power was adjusted to $1 \%$ of its nominal output power and each spectrum was collected with 3 accumulations.

\section{Conductivity measurements under mechanical stretching}

The conductivity of the prepared stretchable electrodes was determined under extreme conditions, which were applied using a universal testing machine (Zwick GmbH \& Co., model Z2.5/TN1S) with integrated testing software (testXpert, Zwick). Electrical conductivities $(\sigma)$ were determined for the stretched specimens using the sheet-resistance method following a previously described procedure. ${ }^{43}$ 


\section{Results and Discussion}

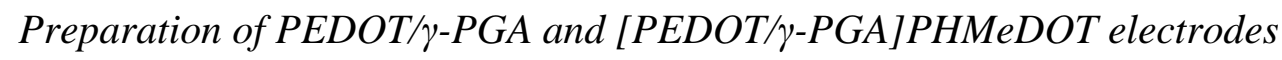

In this work, the synthesis of PEDOT/ $\gamma$-PGA and [PEDOT/ $\gamma$-PGA]PHMeDOT electrodes to be applied in energy storage devices are reported for the first time. PEDOT particles were obtained by applying a sonication treatment to electropolymerized PEDOT films. The influence of the sonication time $\left(t_{\mathrm{son}}\right)$ in the diameter of the resulting particles $\left(D_{\mathrm{CP}}\right)$ was evaluated by DLS in $0.5 \mathrm{M} \mathrm{NaHCO}_{3}$ (Figure 1a). Results indicate that $D_{\text {PEDOT }}$ decrease with increasing $t_{\text {son, }}$, becoming stable at $D_{\text {PEDOT }}=0.85 \pm 0.03 \mu \mathrm{m}$ after $25 \mathrm{~min}$.

In order to achieve a homogenous dispersion of the PEDOT particles in the $\gamma$-PGA matrix, this $D_{\text {PEDOT }}$ value was chosen for the fabrication of PEDOT $/ \gamma$-PGA electrodes. Moreover, the $\gamma$ PGA hydrogel, which was prepared as described in the Methods section, exhibited pores / void spaces with diameters higher than $D_{\text {PEDOT }}$ values (see below). Figure $1 \mathrm{~b}$ shows that the consistency and toughness of the synthesized $\gamma$-PGA hydrogel are suitable to hold the PEDOT particles and to ensure the robustness of the resulting electrode. PEDOT/ $\gamma$-PGA electrodes were prepared by introducing PEDOT particles $(20 \% \mathrm{w} / \mathrm{w})$ in the reaction medium used to cross-link the biopolymer chains. The optical image displayed in Figure 1c shows that the dispersion of the $\mathrm{CP}$ in the $\gamma$-PGA network was good and homogeneous, the final PEDOT $/ \gamma$-PGA electrode exhibiting a blackish appearance. Figure 1d also proves the consistency and flexibility of the PEDOT-loaded hydrogel, which compresses more than $50 \%$ of its initial volume.

On the other hand, the influence of the loaded CP particles in the conductivity of the hydrogel was examined by performing EIS measurements on $\gamma$-PGA and PEDOT/ $\gamma$-PGA using a

cell with a geometry explicitly constructed for the analysis of these polymeric systems. ${ }^{44}$ The 
diameter of the semicircle in the recorded Nyquist plots (not shown) corresponded to the chargetransfer resistance, $R_{\mathrm{C}}$, the conductivity $(\sigma$, in $\mathrm{S} / \mathrm{cm}$ ) being determined through the following expression:

$$
\sigma=\frac{L}{R_{C} A}
$$

where $L$ is the thickness of the coating $(0.02 \mathrm{~cm}), A$ is the area of the coated electrode $\left(1.77 \mathrm{~cm}^{2}\right)$, and $R_{C}$ is the hydrogel resistance. As expected, the incorporation of $20 \% \mathrm{w} / \mathrm{w}$ PEDOT particles significantly affects the electrical conductivity, which increases from $\sigma=6.84 \cdot 10^{-6} \mathrm{~S} / \mathrm{cm}$ for $\gamma$ PGA to $\sigma=1.77 \cdot 10^{-3} \mathrm{~S} / \mathrm{cm}$ for PEDOT $/ \gamma-\mathrm{PGA}$. The latter value is within the typical range attributed to semiconducting materials.

In order to enhance the electrochemical behavior of the electrode, HMeDOT monomers in aqueous solution were anodically polymerized directly inside the loaded PEDOT $/ \gamma$-PGA hydrogel. PHMeDOT was chosen for the electrochemical modification of PEDOT/ $\gamma-\mathrm{PGA}$ because of the following two reasons: ${ }^{45,46}$ (i) the solubility in water of HMeDOT is high, especially when compared with the EDOT monomer, due to the exocyclic hydroxymethyl group; and (ii) the capacitance and electrochemical activity of PEDOT and PHMeDOT are very similar. Accordingly, the exocyclic hydroxymethyl group of HMeDOT allowed us to ensure the success of the polymerization process in water without cause detriment in the electrochemical characteristics of the final [PEDOT/ $\gamma-\mathrm{PGA}] \mathrm{PHMeDOT}$ electrode. It should be emphasized that PEDOT/ $\gamma$-PGA was kept under stirring in the reaction medium overnight (see Methods section). This simple procedure guarantees the penetration of the HMeDOT monomers into the hydrogel matrix ensuring the success of the polymerization process. 
Figure 2a represents the chronoamperograms obtained after the electropolymerization of a 10 mM HMeDOT aqueous solution with $0.1 \mathrm{M} \mathrm{LiClO}_{4}$ as supporting electrolyte on a steel electrode coated with PEDOT $/ \gamma$-PGA and unloaded $\gamma$-PGA hydrogels. For the sake of comparison, the chronoamperometric curve obtained in aqueous solution with $0.1 \mathrm{M} \mathrm{LiClO}_{4}$ (i.e. without HMeDOT monomer) for a steel electrode coated with unloaded $\gamma$-PGA (blank sample) is also reported. As it can be seen, the electropolymerization charge was $25 \%$ higher in the presence of PEDOT particles (i.e. 0.128 and $0.160 \mathrm{C}$ for unloaded $\gamma$-PGA and PEDOT $/ \gamma$-PGA, respectively). This is a very remarkable difference considering that the $\gamma$-PGA hydrogel tends to behave as a dielectric material. Therefore, the presence of PEDOT particles inside the $\gamma$-PGA matrix presumably provides additional nucleation sites that enhance the HMeDOT polymerization.

In order to better understand the polymerization reaction when the hydrogel is part of the reaction medium, a detailed study of the kinetics was conducted. The evolution of current with time during the anodic polymerization of PEDOT, and by extrapolation of PHMeDOT, was explained through three main steps: ${ }^{47}(i)$ the initial spike, which is due to the charging of the double layer; (ii) the region that exhibits a slow variation of the current, which is associated to the CP nucleation; and (iii) the zone in which the current keeps constant over time because of the growth of the polymer chains. However, chronoamperograms displayed in Figure 2a reflect a different current decay during the second step, independently of the absence or presence of PEDOT particles. Specifically, this step ends at $\sim 85 \mathrm{~s}$ and $\sim 105 \mathrm{~s}$ for the polymerization inside PEDOT $/ \gamma$-PGA and unloaded $\gamma$-PGA, respectively. Accordingly, the nucleation of PHMeDOT is most probably affected by the presence of PEDOT microparticles embedded into the hydrogel, which provide charges and radicals able to bind HMeDOT monomers. 
The Cottrell equation was applied using the data shown in Figure 2a to study the diffusion of the HMeDOT molecules in the solution. Figure $2 \mathrm{~b}$ represents the variation of the anodic current density against the inverse of the square of electropolymerization time $\left(\theta^{1 / 2}\right)$. The diffusion coefficient $(D)$ was derived from:

$$
I=\frac{n \cdot F \cdot A \cdot C^{*} \cdot D^{1 / 2}}{(\pi \cdot \theta)^{1 / 2}}
$$

where, $I$ is the anodic current, $C^{*}$ is the molar concentration $(10 \mathrm{mM}), F$ is the Faraday constant, $A$ is the area of the electrode and $n$ is the number of electrons transferred. The value of the current obtained for the blank sample (i.e. electrode coated with unloaded $\gamma$-PGA and without monomer in the reaction medium), which is related to the electron transfer at the steel electrode when the $\mathrm{ClO}_{4}^{-}$molecules reach the surface, was subtracted from the currents recorded during the experiments with PEDOT $/ \gamma$-PGA and unloaded $\gamma$-PGA hydrogels in presence of monomer.

Although Figure $2 \mathrm{~b}$ shows the current decay, a steady state is detected at long times in presence of PEDOT particles. From that, it can be inferred a "transition time" after which the current profile behaves linearly until the end of the experiment. The transition time is defined by the intersection of the two straight lines in Figure 2b, representing ideal transient and steady-state conditions, respectively. According to the Nernst-Planck equation, the flux of species is due to the concentration gradient and the electric field. However, since the $y$-intercept of Cottrell plots is very low (i.e. 0.0013 and $0.022 \mathrm{~mA} \mathrm{~cm}{ }^{2}$ for PEDOT/ $\gamma$-PGA and unloaded $\gamma$-PGA, respectively), it can be considered that the influence of the electric field can be roughly disregarded in both cases. Based on this, the results indicate that the transport of molecules inside the swelled $\gamma$-PGA hydrogel is driven by the concentration gradient of the charge on the infinite-diffusion system. 
According to the Cottrell equation (Eqn 6), the diffusion coefficients were estimated as $4.525 \times 10^{-}$

${ }^{9}$ and $1.739 \times 10^{-9} \mathrm{~cm}^{2} / \mathrm{s}$ for unloaded $\gamma$-PGA and PEDOT/ $\gamma$-PGA hydrogels, respectively. These values, which are significantly higher than those typically expected for substances in solution. Thus, the difficulties in the mobility of HMeDOT molecules inside the hydrogel limit the electrogeneration process. However, the differences between the two systems confirm that PEDOT particles dispersed in the hydrogel matrix act as reaction nuclei, decreasing the diffusion coefficient of the intercepted HMeDOT monomer molecules that bind to PEDOT particles before reaching the steel surface.

On the other hand, the electropolymerization times used in this work were $\theta=6 \mathrm{~min}$ and 7 hours. Considering the current productivity of PHMeDOT in water and the charge consumed during the electropolymerization process inside PEDOT/ $\gamma$-PGA hydrogel (i.e. 1.80 and $33.5 \mathrm{mC}$ for $\theta=6 \mathrm{~min}$ and 7 hours, respectively), the mass of PHMeDOT produced per $1 \mathrm{~mm}^{3}$ of loaded hydrogel is estimated to be around $5 \cdot 10^{-5}$ and $1 \cdot 10^{-3} \mathrm{mg}$ for $\theta=6 \mathrm{~min}$ and 7 hours.

\section{Morphological and topographical analysis}

Figure 3 compares SEM micrographs of $\gamma$-PGA and PEDOT $/ \gamma$-PGA hydrogels before (Figures 3a-b and 3c-f, respectively) and after (Figures 3e and 3f, respectively) CV analyses. As one of the most important objectives of this work is to promote the movement of ions through the flexible electrode, the hydrogel should present an open porous structure and mechanical integrity. Figures 3a-b show the porous structure of the $\gamma$-PGA hydrogel, which exhibits irregularly shaped pores with diameter typically comprised between 2 and $12 \mu \mathrm{m}$ (Figure 3b). A similar structure is observed for PEDOT $/ \gamma$-PGA (Figures 3c-d), even though in this case aggregates of PEDOT 
microparticles dispersed into the biopolymeric matrix are observed (Figure 3d). The dispersion of the PEDOT particles in biopolymeric matrix is reflected in the cross section SEM images of PEDOT/ $\gamma$-PGA (Figures 3e-f), which prove that such particles are not only located at the surface of the $\gamma$-PGA hydrogel but also embedded inside.

After electrochemical analyses, which were performed in water with $0.1 \mathrm{M}$ of $\mathrm{LiClO}_{4}$ as supporting electrolyte, the surface morphology of both $\gamma$-PGA and PEDOT $/ \gamma$-PGA electrodes experienced significant changes (Figures $3 \mathrm{~g}$ and $3 \mathrm{~h}$, respectively). More specifically, the porous hydrogel matrix transforms into a closed structure, while PEDOT particles becomes more compact. These morphological alterations are due to the effects of voltammetric processes on the $\gamma$-PGA and PEDOT chains. More specifically, fast rearrangements of the $\gamma$-PGA chains after the ions transport are possible because of the chemical nature of the crosslinked polymer network. Thus, cystamine offers relatively flexible crosslinks due to the presence of a bridge with four methylene units, providing mobility to the $\gamma$-PGA chains. It should be noted that chain rearrangements are necessary to obtain both a high diffusion rate of the water molecules and a good distribution of the ions inside the matrix. Besides, electrostatic repulsions among the carboxylate groups in the dehydrated structures used for SEM analyses are expected to be significantly mitigated by the presence of $\mathrm{Li}^{+}$ions inside the matrix, which chelate with such negatively charges groups. ${ }^{48}$ In addition, for the PEDOT/ $\gamma$-PGA electrode, at potentials higher than the oxidation potential of the $\mathrm{CP}$, the repulsive forces between emerging positive charges on closer PEDOT chains (i.e. formed polarons) induce conformational movements that generate free volume, facilitating the entrance of counterions and solvent molecules from the solution. In opposition, during the voltammetric reduction, the $\mathrm{CP}$ polymer shrinks since counterions and 
solvent molecules are expelled towards the solution and the structure becomes closed (i.e. interchain distances are shorter than counterion diameters). The influence of the voltammetric oxidation and reduction on PEDOT was studied in detail by Otero and co-workers. ${ }^{49}$

Figure 4 compares SEM micrographs of $\gamma$-PGA after electropolymerization of PHMeDOT during $6 \min$ (Figure 4a), denoted $[\gamma-\mathrm{PGA}] \mathrm{PHMeDOT}(\theta=6 \mathrm{~min})$, and [PEDOT $/ \gamma-$ PGA]PHMeDOT considering electropolymerization times of $\theta=6 \mathrm{~min}$ and 7 hours (Figures $4 \mathrm{~b}$ and $4 \mathrm{c}$, respectively). In absence of PEDOT particles, at low electropolymerization time, no evidence of PHMeDOT is detected on the surface of $\gamma$-PGA, the morphology of $[\gamma$ $\operatorname{PGA}] \operatorname{PHMeDOT}(\theta=6 \mathrm{~min})$ (Figure $4 \mathrm{a})$ being practically identical to that obtained for pure $\gamma$ PGA after apply a voltammetric cycle (Figure 3g). In contrast, the surface of $[\mathrm{PEDOT} / \gamma$ $\operatorname{PGA}] \operatorname{PHMeDOT}(\theta=6 \mathrm{~min})$ is covered by a layer (Figure $4 \mathrm{~b}$ ), which has been attributed to PHMeDOT. Moreover, PEDOT particles are surrounded by ramifications. This feature indicates that the electropolymerization of PHMeDOT starts at the CP particles, corroborating the role of PEDOT particles as nuclei for the electropolymerization process. However, the morphology of PHMeDOT experiences drastic changes when the electropolymerization time increases to $\theta=7 \mathrm{~h}$. More specifically, Figure 4c displays a quite uniform and well-distributed 3D microstructure that densely covers the $\gamma$-PGA surface. As observed, apparently such microstructure results from the assembly of lamellar architectures, suggesting that PHMeDOT organizes according to a foldedchain model. ${ }^{50}$ Moreover, SEM cross section images (Figure 4d) demonstrate that the conductive PHMeDOT networks extends inside the hydrogel matrix.

Representative 3D topographic and 2D phase AFM images of [PEDOT/ $\gamma$ $\operatorname{PGA}] \operatorname{PHMeDOT}(\theta=7 \mathrm{~h})$, which are displayed in Figures $4 \mathrm{e}$ and $4 \mathrm{f}$, respectively, are fully 
consistent with the conclusions extracted from SEM micrographs (Figure 4c). Moreover, AFM images indicate that the observed 3D microstructure presents a very high roughness with $\mathrm{R}_{\mathrm{q}}=783$ nm. Besides, EDX analyses from the sample displayed in Figure 4c confirm the presence of PHMeDOT, as is evidenced by the well-defined sulfur peak (Figure 4g).

\section{Chemical characterization, thermal stability and swelling}

Figure 5a shows the FTIR spectra of $\gamma$-PGA, PEDOT $/ \gamma-$ PGA and $[\mathrm{PEDOT} / \gamma-$ $\operatorname{PGA}] \operatorname{PHMeDOT}(\theta=6 \mathrm{~min}$ and $7 \mathrm{~h})$. The typical absorption bands of the $\gamma$-PGA hydrogel, as identified by Pérez-Madrigal et al., ${ }^{25}$ are detected for all samples. The success of the crosslinking process was proved by comparing with the FTIR spectrum of the biopolymer acquired before the reaction with the cystamine (not shown). Thus, the formation of $-\mathrm{CONH}-$ bonds due to the reaction between the biopolymer and the cross-linker was evidenced by the disappearance of the free carboxylic acid $\left(1718 \mathrm{~cm}^{-1}\right)$ and asymmetric $\mathrm{COO}^{-}$bands $\left(1595 \mathrm{~cm}^{-1}\right)$, and by the enhancement of the amide I $\left(1621 \mathrm{~cm}^{-1}\right)$ and amide II $\left(1530 \mathrm{~cm}^{-1}\right)$, as compared with noncrosslinked $\gamma$-PGA.

Besides, the presence of PEDOT particles in PEDOT $/ \gamma$-PGA is disclosed by the appearance of the $\mathrm{C}-\mathrm{O}-\mathrm{C}$ stretching vibration $\left(1090 \mathrm{~cm}^{-1}\right)$ and ethylendioxy stretching $\left(1180 \mathrm{~cm}^{-1}\right)$ bands. Electropolymerization of PHMeDOT results in the enhancement of such peaks. Further, the broad peak at around $3298 \mathrm{~cm}^{-1}$, which is due to the overlap of $\mathrm{N}-\mathrm{H}$ and $\mathrm{O}-\mathrm{H}$ stretching vibrations from $\gamma$-PGA, experiences a shift towards $3258 \mathrm{~cm}^{-1}$ for the [PEDOT/ $\gamma$ $\operatorname{PGA}] \operatorname{PHMeDOT}(\theta=7 \mathrm{~h})$ sample. Both the blue shift and the narrowing of such peak have been attributed to the high amount of hydroxyl groups arising from PHMeDOT. Moreover, peaks at 
1530, 1575 and $1729 \mathrm{~cm}^{-1}$, which appear using $\theta=7 \mathrm{~h}$, have been associated to enhanced oxidation processes.

Furthermore, Raman spectra and microscopy images were taken from the cross section of transversely cut PEDOT/ $\gamma$-PGA and [PEDOT $/ \gamma-\mathrm{PGA}] \mathrm{PHMeDOT}(\theta=7 \mathrm{~h})$ samples using confocal Raman microscope. Spectra were taken inside the areas marked in images displayed in Figures $5 \mathrm{~b}$ and 5c. As it can be seen, black spots ascribable to the PEDOT particles are clearly identified in Figure 5b, whereas these spots are much less evident in Figure 5c due to the great amount of PHMeDOT covering PEDOT particles.

The spectra reported in Figure $5 \mathrm{~d}$ exhibit the characteristics peaks of PEDOT: $983 \mathrm{~cm}^{-1}$ (vibration mode of the thiophene $\mathrm{C}-\mathrm{S}$ bond), $1085 \mathrm{~cm}^{-1}$ (stretching of the ethylendioxy group), $1255 \mathrm{~cm}^{-1}$ (C-C inter-ring stretching), $1365 \mathrm{~cm}^{-1}$ (C-C stretching), $1430 \mathrm{~cm}^{-1}$ (C=C symmetrical stretching) and $1485 \mathrm{~cm}^{-1}(\mathrm{C}=\mathrm{C}$ asymmetrical stretching). In presence of PHMeDOT, the peaks at 1430 and $1485 \mathrm{~cm}^{-1}$ shift to 1433 and $1496 \mathrm{~cm}^{-1}$, respectively, and, in addition, the intensity of the latter experiences a significant increment. However, the most important feature is the appearance of two new peaks at 2878 and $2960 \mathrm{~cm}^{-1}$, which have been associated to the exocyclic hydroxyl group. Therefore, we conclude that both the loaded PEDOT particles and the PHMeDOT electropolymerized on PEDOT $/ \gamma$-PGA are well and homogenously distributed inside the hydrogel matrix.

The thermal stability is an essential parameter for the potential application of conducting polymers. Unfortunately, the decomposition temperature of these materials is usually low, as is reflected in the literature. ${ }^{51-53}$ Figure 6, which compares the thermogravimetric curves for $\gamma$-PGA, PEDOT $/ \gamma$-PGA and [PEDOT/ $\gamma$-PGA]PHMeDOT $(\theta=7 \mathrm{~h})$, shows a small weight loss (i.e. $10 \%)$ 
at about $100{ }^{\circ} \mathrm{C}$ for $\gamma$-PGA and PEDOT/ $\gamma$-PGA. This loss corresponds to the evaporation of absorbed traces of water. A similar weight loss is detected for [PEDOT/ $\gamma$-PGA]PHMeDOT $(\theta=7$ h) although the evaporation process takes place in a wider temperature interval (i.e. the DTGA peak moves to $122^{\circ} \mathrm{C}$ ), suggesting that the hydrogel network becomes more compact as expected from the performed polymerization.

Figure 6 also indicates that the thermal degradation process is roughly similar for the three samples taking into account that the predominant decomposition step took place around $275^{\circ} \mathrm{C}$. However, detailed analyses reveal some important differences among the three samples. Thus, $\gamma$ PGA shows a multistep degradation that could be justified by the complex molecular architecture that involves different units (e.g. $\gamma$-PGA and the cystamine crosslinker) and also to a non-highly homogeneous matrix. The degradation process becomes more complex for PEDOT/ $\gamma$-PGA in the $240-327^{\circ} \mathrm{C}$ interval as evidenced by the higher number of peaks surrounding the one associated to the maximal decomposition. This feature suggests a hindered diffusion of degradation products caused by the presence of PEDOT particles. Small shoulders at $225^{\circ} \mathrm{C}$ and $350{ }^{\circ} \mathrm{C}$ could also be detected in the DTGA curve as well as a peak at $585^{\circ} \mathrm{C}$. Interestingly, analysis of the DTGA curve obtained for $[\operatorname{PEDOT} / \gamma-\operatorname{PGA}] \operatorname{PHMeDOT}(\theta=7 \mathrm{~h})$ indicates that the polymerization of PHMeDOT inside the hydrogel matrix gives rise to a highly uniform matrix that causes a similar diffusion of degraded molecules and therefore a single predominant peak. Shoulders detected in the PEDOT/ $\gamma$-PGA sample are logically still observed as well as a high temperature peak at 582 ${ }^{\circ} \mathrm{C}$.

The swelling behavior of the different systems, which was determined by gravimetric measurements, is displayed in Table 1. As it can be seen, the swelling ratio of the hydrogels 
increases with the content of CP, growing from $S R=54 \%$ for the pristine $\gamma$-PGA hydrogel to $S R=$ $289 \%$ [PEDOT $/ \gamma$-PGA]PHMeDOT $(\theta=7 \mathrm{~h})$. This effect has been attributed to the hydrophilicity of PEDOT and, specially, of PHMeDOT.

\section{Electrochemical properties}

The electroactivity, specific capacitance (SC) and electrochemical stability of the prepared electrodes were determined by CVs in water with $0.1 \mathrm{M} \mathrm{LiClO}_{4}$. Figure 7 a compares the control voltammograms recorded for $\gamma$-PGA, PEDOT $/ \gamma$-PGA and [PEDOT $/ \gamma$-PGA]PHMeDOT( $\theta=6 \mathrm{~min}$ and $7 \mathrm{~h}$ ). The response of the $\gamma$-PGA hydrogel is more pronounced than that expected for a dielectric. However, previous studies devoted to investigate the electrochemical response of nanomembranes prepared by spin-coating mixtures of polythiophene and insulating thermoplastics ${ }^{54,55}$ proved the electrochemical response of control nanomembranes made with the latter. In spite of this, voltammograms recorded for insulating thermoplastics did not show well-defined oxidation and reduction peaks in water with $\mathrm{LiClO}_{4}$, which was consistent with the formation of charged species at unspecific positions. ${ }^{54,55}$ This represents a significant difference with respect to the voltammogram obtained for the $\gamma$-PGA hydrogel (Figure 7a). In this case, the oxidation peaks with anodic peak potentials of -0.2 and $0.7 \mathrm{~V}$ have been attributed to the formation of irreversible polarons and bipolarons, respectively, at preferred positions. Furthermore, the cathodic scan shows a reduction peak with cathodic peak potential of $0.08 \mathrm{~V}$, which has been attributed to the electrochemical reduction of the amide bond to secondary amine (i.e. electrochemical deoxygenation process). ${ }^{56}$ On the other hand, the electrochemical activity of the $\gamma$-PGA hydrogel is higher than that observed for insulating thermoplastics. ${ }^{54,55}$ This has been 
attributed to the pores (Figure 3) formed by cross-linked polymer chains, which facilitate considerably the access of the electrolyte ions to the surface of the steel substrate.

On the other hand, the electrochemical response obtained for PEDOT/ $\gamma-\mathrm{PGA}$ and [PEDOT/ $\gamma$ $\operatorname{PGA}] \operatorname{PHMeDOT}(\theta=6 \mathrm{~min})$ is apparently similar to that observed for the pristine hydrogel (Figure 7a, inset). Thus, the incorporation of $\mathrm{CP}$ inside the dielectric hydrogel matrix does not cause an increment in the electrochemical activity, even though the oxidation and reduction of PEDOT and PHMeDOT are typically detected. ${ }^{37}$ The first oxidation peak of chains occurs at 0.5 $\mathrm{V}$, while the second peak overlaps with the oxidation potential of the medium. In addition, two reduction peaks are detected in the cathodic scans, indicating the presence of redox pairs in the recorded potential range. These redox processes, which are clearly identified for both PEDOT/ $\gamma$ PGA and $[\mathrm{PEDOT} / \gamma-\mathrm{PGA}] \mathrm{PHMeDOT}(\theta=6 \mathrm{~min})$, should be attributed to the formation of polarons in the CP chains.

The electroactivities, which were quantified as the voltammetric stored charge per surface unit $(Q)$, and the areal specific capacitances $(S C$, Eqn 1) of $\gamma$-PGA, PEDOT/ $\gamma$-PGA and $[\operatorname{PEDOT} / \gamma-\operatorname{PGA}] \operatorname{PHMeDOT}(\theta=6 \mathrm{~min})$, are listed in Table 1 . Consistently with the voltammograms displayed in Figure 7a, the electrochemical properties of the three systems were lower than those typically obtained for CPs. ${ }^{53,57}$ More specifically, the values of $Q$ and $S C$ determined for PEDOT films prepared using identical experimental conditions are $4.25 \cdot 10^{-2}$ $\mathrm{C} / \mathrm{cm}^{2}$ and $27 \mathrm{mF} / \mathrm{cm}^{2}$, respectively.

Enlargement of the PHMeDOT polymerization time from $\theta=6 \min$ to $t=7 \mathrm{~h}$ causes a remarkable improvement of the electrochemical properties, which is reflected by the gain in both the cathodic and anodic areas of the recorded voltammogram (Figure 7a). The electroactivity is 
one order of magnitude higher for $[\mathrm{PEDOT} / \gamma-\mathrm{PGA}] \mathrm{PHMeDOT}(\theta=7 \mathrm{~h})$ than for the other three systems (Table 1). The well-defined oxidation peak, with anodic peak potencial $\mathrm{E}_{\mathrm{p}}{ }^{\mathrm{a}} \approx 1.35 \mathrm{~V}$ and the reduction shoulder, with cathodic peak potential $\mathrm{E}_{\mathrm{p}}{ }^{\mathrm{c}}=-0.02 \mathrm{~V}$, are consistent with the very high content of PHMeDOT at the electrode and reflect a remarkable redox charge storage capacity. The $S C$ of [PEDOT/ $\gamma-\mathrm{PGA}] \operatorname{PHMeDOT}(\theta=7 \mathrm{~h}), 45.4 \mathrm{mF} / \mathrm{cm}^{2}$, is almost 20 times higher than the areal capacitance of PEDOT/ $\gamma$-PGA $\left(2.3 \mathrm{mF} / \mathrm{cm}^{2}\right)$, and several times higher than those recently reported for other flexible PEDOT-based organic electrodes, as for example: fourlayered PEDOT:poly(styrene sulfonate) (PSS) films $\left(4.7 \mathrm{mF} / \mathrm{cm}^{2}\right),{ }^{58}$ graphene oxide/PEDOT and reduced graphene oxide/PEDOT composites electropolymerized onto flexible substrates (16 and $25 \mathrm{mF} / \mathrm{cm}^{2}$, respectively), ${ }^{59}$ and PEDOT electrochemically deposited on PEDOT:PSS/cellulose substrates (from 11 to $\left.32 \mathrm{mF} / \mathrm{cm}^{2}\right) .{ }^{60}$ Moreover, the $S C$ of $[\operatorname{PEDOT} / \gamma-\operatorname{PGA}] \operatorname{PHMeDOT}(\theta=7 \mathrm{~h})$ experienced a slight increment after a few consecutive oxidation-reduction cycles (i.e. from 45.4 to $46.1 \mathrm{mF} / \mathrm{cm}^{2}$ after ten cycles), suggesting that such electrochemical processes induce small structural rearrangements that favor the interactions between different chemical components. This synergistic interaction was not observed for PEDOT $/ \gamma-\mathrm{PGA}$ and $[\mathrm{PEDOT} / \gamma-\mathrm{PGA}] \mathrm{PHMeDOT}(\theta=$ $6 \mathrm{~min})$.

The lifetime stability of [PEDOT/ $\gamma$-PGA]PHMeDOT $(\theta=7 \mathrm{~h})$ was examined by submitting this electrode to one-thousand consecutive GCD cycles from -0.50 to $0.40 \mathrm{~V}$ at a current of 0.1 mA (Figures 7b). GCD curves exhibited slight distortions with somewhat non-symmetrical curves since the discharge time was imposed to be lower than the charge time. The areal $S C$ of the PEDOT/ $\gamma$-PGA]PHMeDOT( $\theta=7 \mathrm{~h})$ electrode, as derived from Eqn $3\left(47.2 \mathrm{mF} / \mathrm{cm}^{2}\right)$, showed an outstanding retention of $87.5 \%$ after 1000 cycles $\left(41.3 \mathrm{mF} / \mathrm{cm}^{2}\right)$. Besides, the mechanical 
characteristics of the $[\mathrm{PEDOT} / \gamma-\mathrm{PGA}] \mathrm{PHMeDOT}(\theta=7 \mathrm{~h})$ electrode remains practically unaltered after multiple charge-discharge cycles. This is demonstrated in Figure $7 \mathrm{c}$, which shows the robustness and compression behavior of the electrode after the 1000 GCD cycles. Specifically, the electrode can be compressed by more than $50 \%$ without signs of damages.

\section{Practical applications: Conductivity changes under stretching and powering a LED bulb}

The $[\mathrm{PEDOT} / \gamma-\mathrm{PGA}] \mathrm{PHMeDOT}(\theta=7 \mathrm{~h})$ electrode retains the flexibility and compression behavior of the $\gamma$-PGA hydrogel. In recent years the importance of organic flexible and stretchable electrodes has been reviewed different authors. ${ }^{61-64}$ Important advances have been also described for PEDOT-based electrodes. For example, Cheng et al. ${ }^{65}$ reported flexible transparent electrodes with very good electrochemical and optoelectronic performance by combining Ag grids with PEDOT:poly(styrene sulfonate) (PSS) layers on polyethylene terephthalate substrates using an inkjet printing methodology. The electromechanical properties of these electrodes were superior to those achieved for flexible electrodes constructed embedding Ag nanowires into poly(dimethylsiloxane). ${ }^{68}$ Kurungot and co-workers ${ }^{66}$ prepared highly conducting and robust PEDOT-paper electrodes using a surfactant-free interfacial polymerization at the interface of two immiscible liquids. This procedure resulted in flexible PEDOT films with highly ordered polymer chains and enhanced doping level. Liu et al. obtained highly flexible, bendable and conductive graphene-PEDOT:PSS films using a simple bar-coating method. The assembled device using rGO-PEDOT/PSS electrode could be bent and rolled up without any decrease in electrochemical performance. ${ }^{67}$ 
Measurements of conductivity changes have been made in flexible [PEDOT/ $\gamma$ $\operatorname{PGA}] \operatorname{PHMeDOT}(\theta=7 \mathrm{~h})$ electrode when subjected to a uniaxial strain of up to $10 \%$ (Figure $8 \mathrm{a}$ ). For this purpose, the prepared specimens, which consisted of rectangular sheets of $30 \mathrm{~mm}$ (length) $\times 5 \mathrm{~mm}$ (width) $\times 0.8 \mathrm{~mm}$ (thickness), were stretched at a rate of $2 \mathrm{~mm} / \mathrm{min}$. Figure $8 \mathrm{~b}$ displays the change in resistance under tensile deformation. Although the conductivity decreases linearly with increasing uniaxial strain, changes are relatively small (37\% for the maximum tensile deformation). These results indicate that embedded $\mathrm{CP}$ regions remain relatively associated among them and, therefore, the electrode's electrical properties experience relatively small variations. Unfortunately, the situation changes when the uniaxial strain is $\geq 15 \%$. In that case, the electrical resistivity increases drastically, indicating the irreversible dissociation of conductive regions inside the hydrogel. This should not be attributed to the rupture of the hydrogel but to the very poor mechanical properties of PHMeDOT and PEDOT particles, which are not able to follow the elastic movements of $\gamma$-PGA chains without damage.

Finally, the $[\mathrm{PEDOT} / \gamma-\mathrm{PGA}] \mathrm{PHMeDOT}(\theta=7 \mathrm{~h})$ electrode displayed in Figure $8 \mathrm{c}$ was used in a simple demonstration of an energy harvesting system by powering a red LED bulb. For this purpose, a Teflon holder with a stainless steel (AISI 304) disk was assembled with the $[\mathrm{PEDOT} / \gamma-\mathrm{PGA}] \mathrm{PHMeDOT}(\theta=7 \mathrm{~h})$ electrode, as is illustrated in Figure $8 \mathrm{~d}$. The assembled system was charged by coupling a power supply of $24 \mathrm{~V}$ and a resistance of $20 \mathrm{k} \Omega$ (Figure 8e). Such two elements were retired after complete the recharge, employing about $60 \mathrm{~s}$. As the voltage required to power a LED bulb is about $1.5 \mathrm{~V}$, the system was connected in series (Figure $8 \mathrm{f}$ ) with the LED bulb to get an open circuit voltage of $2.2 \mathrm{~V}$. The LED bulb was powered during intervals of time of $\sim 125 \mathrm{~s}$, the discharge through the powering of the red LED being displayed in 
Figure $8 \mathrm{~g}$. This result corroborates that the $[\mathrm{PEDOT} / \gamma-\mathrm{PGA}] \mathrm{PHMeDOT}$ electrode composite is able to supply enough current instantaneously, evidencing its applicability in flexible energyharvesting systems (this work is currently in progress).

\section{Conclusions}

Highly flexible and lightweight free-standing electrodes have been synthesized by functionalizing $\gamma$-PGA hydrogels with PEDOT particles, which were subsequently used as polymerization nuclei for the anodic polymerization of PHMeDOT. The $\gamma$-PGA hydrogel provides a support with consistency, robustness and open internal structure, which is crucial to permit the ion diffusion process. PEDOT particles play a key role in the electropolymerization of HMeDOT monomer, favoring the homogeneous distribution of PHMeDOT chains across the hydrogel. The resulting $[\mathrm{PEDOT} / \gamma-\mathrm{PGA}] \operatorname{PHMeDOT}(\theta=7 \mathrm{~h})$ composite presents a great potential in supercapacitors with specific capacitance hitting $45-47 \mathrm{mF} / \mathrm{cm}^{2}$, as obtained by $\mathrm{CV}$ and GCD, and excellent cycle durability. The effectiveness of this electrode has been proved through a simple application based on power a red LED. The as-made [PEDOT/ $\gamma-\mathrm{PGA}] \mathrm{PHMeDOT}(\theta=7 \mathrm{~h})$ electrodes can be potentially used in various fields, as for example textiles (e.g. wearable electronics) and biomedic, where robustness and flexibility is required.

\section{Acknowledgments}

Authors thank supports from MINECO and FEDER (MAT2015-69367-R and MAT201569547-R). Support for the research of C.A. was received through the prize "ICREA Academia" for excellence in research funded by the Generalitat de Catalunya. 


\section{References}

1. Wang, X.; Lu, X.; Liu, B.; Chen, D.; Tong, Y.; Shen, G. Flexible Energy-Storage Devices: Design Consideration and Recent Progress. Adv. Mater. 2014, 26, 4763-4782.

2. Kim, J.; Lee, J.; You, J.; Park, M. S.; Al Hossain, M. S.; Yamauchi, Y.; Kim, J. H. Conductive Polymers for Next-Generation Energy Storage Systems: Recent Progress and New Functions. Mater. Horiz. 2016, 3, 517-535.

3. Rezzak, D.; Boudjerda, N. Management and Control Strategy of a Hybrid Energy Source Fuel Cell/Supercapacitor in Electric Vehicles. Int. Trans. Electr. Energ. Syst. 2017, 27, e2308.

4. Kim, M.-J.; Peng, H. Power Management and Design Optimization of Fuel Cell/Battery Hybrid Vehicles. J. Power Sources 2007, 165, 819-832.

5. Bruce, P. G.; Freunberger, S. A.; Hardwick, L. J.; Tarascon, J.-M. Li- $\mathrm{O}_{2}$ and Li-S Batteries with High Energy Storage. Nature Mater. 2012, 11, 19-29.

6. Harfman-Todorovic, M.; Palma, L.; Enjeti, P. Power Electronics Specialists Conference, 2006. PESC '06. 37th IEEE. 10.1109/pesc.2006.1712101.

7. Gao, W.; Singh, N.; Song, L.; Liu, Z.; Mohana-Reddy, A. L.; Ci, L.; Vajtai, R.; Zhang, Q.; Wei, B.; Ajayan, P. M. Direct Laser Writing of Micro-Supercapacitors on Hydrated Graphite Oxide Films. Nature Nanotech. 2011, 6, 496-500.

8. Guo, H.; Yeh, M.-H.; Zi, Y.; Wen, Z.; Chen, J.; Liu, G.; Hu, C.; Wang, Z. L. Ultralight CutPaper-Based Self-Charging Power Unit for Self-Powered Portable Electronic and Medical Systems. ACS Nano 2017, 11, 4475-4482. 
9. Shao, Y.; El-Kady, M. F.; Lin, C.-W.; Zhu, G.; Marsh, K. L.; Hwang, J. Y.; Zhang, Q.; Li, Y.; Wang, H.; Kane, R. B. 3D Freeze-Casting of Cellular Graphene Films for Ultrahigh-PowerDensity Supercapacitors. Adv. Mater. 2016, 28, 6719-6726.

10. B. E. Conway, Electrochemical Supercapacitors: Scientific Fundamentals and Technological Applications, Kluwer academic/Plenum Publishing, New York, 1999.

11. Pandolfo, A. G.; Hollenkamp, A. F. Carbon Properties and Their Role in Supercapacitors. $J$. Power Sources 2006, 157, 11-27.

12. González, A.; Goikolea, E.; Barrena, J. A.; Mysyk, R. Review on Supercapacitors: Technologies and Materials. Renew. Sustainable Energy Rev. 2016, 58, 1189-1206.

13. Wu, X.-L.; Xu, A.-W. Carbonaceous Hydrogels and Aerogels for Supercapacitors. J. Mater. Chem. A 2014, 2, 4852-4864.

14. Bajaj, I.; Singhal, R. Poly (glutamic acid)--an Emerging Biopolymer of Commercial Interest. Bioresour. Technol. 2011, 102, 5551-5561.

15. Shih, I.; Wu, P.; Shieh, C. Microbial Production of a Poly( $\gamma$-glutamic acid) Derivative by Bacillus Subtilis. Process Biochem. 2005, 40, 2827-2832.

16. Thome, C. B.; Gómez, C. G.; Noyes, H. E.; Housewright, R. D. Production of Glutamyl Polypeptide by Bacillus Subtilis. J. Bacteriol. 1954, 68, 307-315.

17. Bhat, A. R.; Irorere, V. U.; Bartlett, T.; Hill, D.; Kedia, G.; Morris, M. R. ; Charalampopoulos, D.; Radecka, I. Bacillus Subtilis Natto: A Non-Toxic Source of Poly- $\gamma-$ Glutamic Acid that Could be Used as a Cryoprotectant for Probiotic Bacteria. AMB Express 2013, 3,36 . 
18. Bhat, A. R.; Irorere, V. U.; Bartlett, T.; Hill, D.; Kedia, G.; Charalampopoulos, D.; Nualkaekul, S.; Radecka, I. Improving Survival of Probiotic Bacteria Using Bacterial Poly- $\gamma$ glutamic Acid. Int. J. Food Microbiol. 2015, 196, 24-31.

19. Akao, T.; Kimura, T.; Hirofuji, Y.; Matsunaga, K.; Imayoshi, R.; Nagao, J.; Cho, T.; Matsumoto, H.; Ohtono, S.; Ohno, J.; Taniguchi, K.; Kaminishi, H. A. Poly(gamma-glutamic acid)-Amphiphile Complex as a Novel Nanovehicle for Drug Delivery System. J. Drug Targeting 2010, 18, 550-556.

20. Mi, F. L.; Wu, Y. Y.; Lin, Y. H.; Sonaje, K.; Ho, Y. C.; Chen, C. T.; Juang, J. H.; Sung, H. W. Oral Delivery of Peptide Drugs Using Nanoparticles Self-Assembled by Poly( $\gamma$-glutamic acid) and a Chitosan Derivative Functionalized by Trimethylation. Bioconjugate Chem. 2008, 19, $1248-1255$.

21. Zhang, R.; Lin, L.; Xu, S.; Zhang, C.; Liu, X.; Luo, J. Liquid-Liquid Interfacial Behavior of Dopamine Modified Poly( $\gamma$-glutamic acid) Polymer. Colloids Surf. A 2015, 470, 218-223.

22. Poo, H.; Park, C.; Kwak, M.; Choi, D.; Hong, S.; Lee, I.; Lim, Y. T.; Choi, Y. K.; Bae, S.; Uyama, H.; Kim, C.; Sung, M. New Biological Functions and Applications of High-MolecularMass Poly- $\gamma$-glutamic Acid. Chem. Biodiversity 2010, 7, 1555-1562.

23. Yu, X.; Wang, M.; Wang, Q.; Wang, X. Biosynthesis of Polyglutamic Acid and Its Application on Agriculture. Adv. Mat. Res. 2011, 183-185, 1219-1223.

24. Tsao, C. T.; Chang, C. H.; Lin, Y. Y.; Wu, M. F.; Wang, J. L.; Han, J. L.; Hsieh, K. H. Antibacterial Activity and Biocompatibility of a Chitosan-Gamma-Poly(glutamic acid) Polyelectrolyte Complex Hydrogel. Carbohydr. Res. 2010, 345, 1774-1780. 
25. Pérez-Madrigal, M. M.; Edo, M. G.; Díaz, A.; Puiggalí, J.; Alemán, C. Poly- $\gamma$-glutamic Acid Hydrogels as Electrolyte for Poly(3,4-ethylenedioxythiophene)-Based Supercapacitors. J. Phys. Chem. C 2017, 121, 3182-3193.

26. Armelin, E.; Perez-Madrigal, M. M.; Alemán, C.; Díaz-Díaz, D. Current Status and Challenges of Biohydrogels for Applications as Supercapacitors and Secondary Batteries. $J$. Mater. Chem. A, 2016, 4, 8952-8968.

27. Kirchmeyer, S.; Reuter, R. Scientific Importance, Properties and Growing Applications of Poly(3,4-ethylenedioxythiophene). J. Mater. Chem. 2005, 15, 2077-2088.

28. Grenier, C. R. G.; Pisula, W.; Joncheray, T. J.; Müllen, K.; Reynolds, J. R. Regiosymmetric Poly(dialkylphenylenedioxythiophene)s: Electron-Rich, Stackable $\pi$-Conjugated Nanoribbons. Angew. Chem. Int. Ed. 2007, 46, 714-717.

29. Mantione, D.; del Agua, I.; Sanchez-Sanchez, A.; Mecerreyes, D. Poly(3,4ethylenedioxythiophene) (PEDOT) Derivatives: Innovative Conductive Polymers for Bioelectronics. Polymers 2017, 9, 354.

30. Elschner, A.; Kirchmeyer, S.; Lovenich, W.; Merker, U.; Reuter, K. PEDOT: Principles and Applications of an Intrinsically Conductive Polymer, CRC Press, 2010.

31. Poater, J.; Casanovas, J.; Solà, M.; Alemán, C. Examining the Planarity of Poly(3,4ethylenedioxythiophene): Consideration of Self-Rigidification, Electronic, and Geometric Effects. J. Phys. Chem. A 2010, 114, 1023-1028.

32. Lee, D.-Y.; Na, S.-I.; Kim, S.-S. Graphene Oxide/PEDOT:PSS Composite Hole Transport Layer for Efficient and Stable Planar Heterojunction Perovskite Solar Cells. Nanoscale 2016, 8, $1513-1523$. 
33. Wu, X.-L.; Xu, A.-W. Carbonaceous Hydrogels and Aerogels for Supercapacitors. J. Mater. Chem. A 2014, 2, 4852-4864.

34. Nabilah Azman, N. H.; Lim, H. N.; Sulaiman, Y. Effect of Electropolymerization Potential on the Preparation of PEDOT/ Graphene Oxide Hybrid Material for Supercapacitor Application. Electrochim. Acta 2016, 188, 785-792.

35. Dettlaff, A.; Wilamowska, M. Electrochemical Synthesis and Characterization of Nanocomposites Based on Poly(3,4-ethylenedioxythiophene) and Functionalized Carbon Nanotubes. Synth. Met. 2016, 212, 31-43.

36. Yan, L.; Rui, X.; Chen, G.; Xu, W.; Zou, G.; Luo, H. Recent Advances in Nanostructured Nb Based Oxides for Electrochemical Energy Storage Nanoscale 2016, 8, 8443-8465.

37. Saborío, M. C. G.; Estrany, F.; Alemán, C. Properties of In Situ Polymerized Poly(3,4ethylenedioxythiophene)/Alumina Composites for Energy Storage Applications. J. Polym. Sci., Part B: Polym. Phys. 2017, 55, 1131-1141.

38. Pan, L.; Yu, G.; Zhai, D.; Lee, H. R.; Zhao, W.; Liu, N.; Wang, H.; Tee, B. C.-K.; Shi, Y.; Cui, Y.; Bao, Z. Hierarchical Nanostructured Conducting Polymer Hydrogel with High Electrochemical Activity. Proc. Natl. Acad. Sci. U. S. A. 2012, 109, 9287-9292.

39. Ajjan, F. N.; Casado, N.; Rębis', T.; Elfwing, A.; Solin, N.; Mecerreyes, D.; Inganäs, O. Performance PEDOT/Lignin Biopolymer Composites for Electrochemical Supercapacitors. J. Mater. Chem. A 2016, 4, 1838-1847.

40. Fabregat, G.; Teixeira-Dias, B.; del Valle, L. J.; Armelin, E.; Estrany, F.; Alemán, C. Incorporation of a Clot-Binding Peptide into Polythiophene: Properties of Composites for Biomedical Applications. ACS Appl. Mater. Interfaces 2014, 6, 11940-11954. 
41. López-Pérez, D.; Aradilla, D.; del Valle, L. J.; Alemán, C. Capacitive Composites Made of Conducting Polymer and Lysozyme: Toward the Biocondenser. J. Phys. Chem. C 2013, 117, $6607-6619$.

42. Matsusaki, M.; Yoshida, H.; Akashi, M. The Construction of 3D-Engineered Tissues Composed of Cells and Extracellular Matrices by Hydrogel Template Approach. Biomaterials, 2007, 28, 2729-2737.

43. Brillas, E.; Carrasco, J.; Oliver, R.; Estrany, F.; Vilar, J.; Morlans, J. M. Electropolymerization of 2,5-Di-(-2-thienyl)-pyrrole in Ethanolic Medium. Effect of Solution Stirring on Doping with Perchlorate and Chloride Ions. Electrochim. Acta 2000, 45, 4049-4057.

44. Müller, F.; Ferreira, C. A.; Azambuja, D.; Alemán, C.; Armelin, E. New Sulfonated Polystyrene and Styrene-Ethylene/Butylene-Styrene Block Copolymers for Applications in Electrodialysis. J. Phys. Chem. B 2014, 118, 1102-1112.

45. Fabregat, G.; Casanovas, J.; Redondo, E.; Armelin, E.; Alemán, C. A Rational Design for the Selective Detection of Dopamine Using Conducting Polymers. Phys. Chem. Chem. Phys. 2014, $16,7850-7861$.

46. Hocevar, M. A.; Fabregat, G.; Armelin, E.; Ferreira, C. A.; Alemán, C. Nanometric Polythiophene Films with Electrocatalytic Activity for Non-Enzymatic Detection of Glucose. Eur. Polym. J. 2016, 79, 132-139.

47. Patra, S.; Barai, K.; Munichandraiah, N. Scanning Electron Microscopy Studies of PEDOT Prepared by Various Electrochemical Routes. Synth. Metals 2008, 158, 430-435.

48. Li, Z.; He, G.; Hua, J.; Wu, M.; Guo, W.; Gong, J.; Zhang, J.; Qiao, C. Preparation of $\gamma$-PGA Hydrogels and Swelling Behaviors in Salt Solutions with Different Ionic Valence Numbers. RSC Adv. 2017, 7, 11085-11093. 
49. Otero, T. F.; Caballero Romero, M. Conformational Energy from the Oxidation Kinetics of Poly(3,4-ethylenedioxythiophene) Films. Polym. Int. 2010, 59, 329-336.

50. Lattach, Y.; Coletta, C.; Ghosh, S.; Remita, S. Radiation-Induced Synthesis of Nanostructured Conjugated Polymers in Aqueous Solution: Fundamental Effect of Oxidizing Species. Chem.Phys.Chem. 2014, 15, 208-218.

51. Hu, D.; Lu, B.; Duan, X.; Xu, J.; Zhang, L.; Zhang, K.; Zhang, S.; Zhen, S. Synthesis of Novel Chiral L-Leucine Grafted PEDOT Derivatives with Excellent Electrochromic Performances. RSC Adv. 2014, 4, 35597-35608.

52. Sivula, K.; Luscombe, C. K.; Thompson, B. C.; Frechet, J. M. J. Enhancing the Thermal Stability of Polythiophene: Fullerene Solar Cells by Decreasing Effective Polymer Regioregularity. J. Am. Chem. Soc. 2006, 128, 13988-13989.

53. Aradilla, D.; Azambuja, D.; Estrany, F.; Casas, M. T.; Ferreira, C. A.; Alemán, C. Hybrid Polythiophene-Clay Exfoliated Nanocomposites for Ultracapacitor Devices. J. Mater. Chem. 2012, 22, 13110-13122.

54. Pérez Madrigal, M. M.; Armelin, E.; del Valle, L. J.; Estrany, F.; Alemán, C. Bioactive and Electroactive Response of Flexible Polythiophene:Polyester Nanomembranes for Tissue Engineering. Polym. Chem. 2012, 3, 979-991.

55. Pérez-Madrigal, M. M.; Giannotti, M. I.; del Valle, L. J.; Franco, L.; Armelin, E.; Puiggalí, J.; Sanz, F.; Alemán, C. Thermoplastic Polyurethane:Polythiophene Nanomembranes for Biomedical and Biotechnological Applications. ACS Appl. Mater. Interfaces 2014, 6, 9719-9732. 56. Grimshaw, J. In Electrochemical Reactions and Mechanisms in Organic Chemistry, Elsevier, 2000, pp 330-370. 
57. Sánchez-Jiménez, M.; Estrany, F.; Alemán, C. Improving the Fabrication of AllPolythiophene Supercapacitors. Polym. Sci. Series B 2017, 59, 194-201.

58. Cheng, T.; Zhang, Y.-Z.; Zhang, J.-D.; Lai, W.-Y.; Huang, W. High-Performance FreeStanding PEDOT:PSS Electrodes for Flexible and Transparent All-Solid-State Supercapacitors. J. Mater. Chem. A 2016, 4, 10493-10499.

59. Lehtimäki, S.; Suominen, M.; Damlin, P.; Tuukkanen, S.; Kvarnström, C.; Lupo, D. Preparation of Supercapacitors on Flexible Substrates with Electrodeposited PEDOT/Graphene Composites. ACS Appl. Mater. Interfaces 2015, 7, 22137-22147.

60. Kurra, N.; Park, J.; Alshareef, H. N. A Conducting Polymer Nucleation Scheme for Efficient Solid-State Supercapacitors on Paper. J. Mater. Chem. A 2014, 2, 17058-17065.

61. Cheng, T.; Zhang, Y.; Lai, W.-Y.; Huang, W. Stretchable Thin-Film Electrodes for Flexible Electronics with High Deformability and Stretchability. Adv. Mater. 2015, 27, 3349-3376.

62. Zhang, Y.-Z.; Wang, Y.; Cheng, T.; Lai, W.-Y.; Pang, H.; Huang, W. Flexible Supercapacitors Based on Paper Substrates: A New Paradigm For Low-Cost Energy Storage. Chem. Soc. Rev. 2015, 44, 5181-5199.

63. Pérez-Madrigal, M. M.; Edo, M. G.; Alemán, C. Powering the Future: Application of Cellulose-Based Materials for Supercapacitors. Green Chem. 2016, 18, 5930-5956.

64. Xiao, Y.; Hwang, J.-Y.; Sun, Y.-K. Transition Metal Carbide-Based Materials: Synthesis and Applications in Electrochemical Energy Storage. J. Mater. Chem. A 2016, 4, 10379-10393

65. Cheng, T.; Zhang, Y.-Z.; Yi, J.-P.; Yang, L.; Zhang, J.-D.; Lai, W.-Y.; Huang, W. InkjetPrinted Flexible, Transparent and Aesthetic. J. Mater. Chem. A 2016, 4, 13754-13763. 
66. Anothumakkool, B.; Soni, R.; Bhange, S. N.; Kurungot, S. Novel scalable synthesis of highly conducting and robust PEDOT paper for a high performance flexible solid supercapacitor. Energy Environ. Sci. 2015, 8, 1339-1347.

67. Liu, Y.; Weng, B.; Razal, J. M.; Xu, Q.; Zhao, C.; Hou, Y.; Seyedin, S.; Jalili, R.; Wallace, G. G.; Chen, J. High-Performance Flexible All-Solid-State Supercapacitor from Large FreeStanding Graphene-PEDOT/PSS Films. Sci. Rep. 2015, 5, 17045.

68. Cheng, T.; Zhang, Y.-Z.; Lai, W.-Y.; Chen, Y.; Zeng, W.-J.; Huang, W. High-Performance Stretchable Transparent Electrodes Based on Silver Nanowires Synthesized via an Eco-Friendly Halogen-Free Method. J. Mater. Chem. C 2014, 2, 10369-10376. 


\section{CAPTIONS TO FIGURES}

Figure 1. (a) Variation of the average diameter of PEDOT particles ( $D_{\text {PEDOT }}$ ) against the sonication time $\left(t_{\text {son }}\right)$ as revealed by DSL measurements in $0.5 \mathrm{M} \mathrm{NaHCO}_{3}$. Both the average and the standard deviation for each $t_{\text {son }}$ were calculated using the registered population $v s$. diameter distribution profile. Optical images of (b) the unloaded $\gamma$-PGA and (c) the PEDOT/ $\gamma$-PGA hydrogels. (d) Optical images illustrating the consistency and compression behavior of PEDOT/ $\gamma$ PGA.

Figure 2. (a) Chronoamperograms recorded in $0.1 \mathrm{M} \mathrm{LiClO}_{4}$ aqueous solution for unloaded $\gamma$ PGA, unloaded $\gamma$-PGA with $10 \mathrm{mM}$ of HMeDOT monomer, and PEDOT/ $\gamma$-PGA with $10 \mathrm{mM}$ of EDOT-OH monomer. (b) Cottrell plots for unloaded $\gamma$-PGA and PEDOT/ $\gamma$-PGA with $10 \mathrm{mM}$ of HMeDOT monomer.

Figure 3. SEM micrographs of (a, b, e) unloaded $\gamma$-PGA and (c, d, e, f, h) PEDOT/ $\gamma$-PGA hydrogels. Images $(\mathrm{e}, \mathrm{f})$ correspond to the cross section of the PEDOT/ $\gamma$-PGA hydrogel. Images before (a-f) and after (g, h) analysis by $\mathrm{CV}$ in acetonitrile with $0.1 \mathrm{M}$ of $\mathrm{LiClO}_{4}$ are displayed. Additionally, (b), (d) and (f) display high magnification images of (a), (c) and (e), respectively.

Figure 4. Surface SEM micrographs of (a) $[\gamma-\mathrm{PGA}] \operatorname{PHMeDOT}(\theta=6 \mathrm{~min})$, (b) $[\mathrm{PEDOT} / \gamma-$ $\operatorname{PGA}] \operatorname{PHMeDOT}(\theta=6 \mathrm{~min})$, and (c) $[\operatorname{PEDOT} / \gamma-\operatorname{PGA}] \operatorname{PHMeDOT}(\theta=7 \mathrm{~h})$. (d) $\operatorname{Cross} \operatorname{section}$ SEM image of [PEDOT $/ \gamma-\mathrm{PGA}] \operatorname{PHMeDOT}(\theta=7 \mathrm{~h})$. (e) 3D topographic and (f) $2 \mathrm{D}$ phase AFM images of [PEDOT/ $\gamma-\mathrm{PGA}] \operatorname{PHMeDOT}(\theta=7 \mathrm{~h})$. (g) EDX analysis of the sample displayed in (c).

Figure 5. (a) FTIR spectra for pure $\gamma$-PGA, PEDOT $/ \gamma$-PGA, and [PEDOT/ $\gamma$-PGA]PHMeDOT $(\theta=$ 6 min and 7 h). Images obtained using a confocal Raman microscope for (b) PEDOT/ $\gamma$-PGA and 
(c) $[\mathrm{PEDOT} / \gamma-\mathrm{PGA}] \mathrm{PHMeDOT}(\theta=7 \mathrm{~h})$. White squares define the areas used to record de Raman spectra. (d) Raman spectra of $\operatorname{PEDOT} / \gamma-\mathrm{PGA}$ and $[\operatorname{PEDOT} / \gamma-\operatorname{PGA}] \operatorname{PHMeDOT}(\theta=7 \mathrm{~h})$. Excitation wavelength: $785 \mathrm{~nm}$.

Figure 6. Thermogravimetric (solid lines) and derivative thermogravimetric curves (dashed lines) for $\gamma$-PGA, PEDOT $/ \gamma$-PGA and [PEDOT $/ \gamma$-PGA]PHMeDOT $(\theta=7 \mathrm{~h})$. Details of the region associated to the main decomposition process are provided in the inset.

Figure 7. (a) Control voltammograms $\left(2^{\text {nd }}\right.$ cycle $)$ for $\gamma$-PGA, PEDOT $/ \gamma$-PGA, [PEDOT $/ \gamma$ $\operatorname{PGA}] \operatorname{PHMeDOT}(\theta=6 \mathrm{~min})$ and $[\mathrm{PEDOT} / \gamma-\operatorname{PGA}] \operatorname{PHMeDOT}(\theta=7 \mathrm{~h})$. Initial and final potential: -0.50 ; reversal potential: $1.10 \mathrm{~V}$; scan rate of $100 \mathrm{mV} / \mathrm{s}$. (b) Galvanostatic charge-discharge (GCD) curves recorded at $0.1 \mathrm{~mA}$ (charging and discharging times of 30 seconds) for [PEDOT $/ \gamma$ $\operatorname{PGA}] \operatorname{PHMeDOT}(\theta=7 \mathrm{~h})$. The second cycle is displayed at the right. (c) Photographs reflecting the mechanical robustness and compression behavior of $[\mathrm{PEDOT} / \gamma-\mathrm{PGA}] \operatorname{PHMeDOT}(\theta=7 \mathrm{~h})$ after $1000 \mathrm{GCD}$ cycles.

Figure 8. (a) Photographs showing the electrical conductivity measurement under stretching conditions of the flexible [PEDOT $/ \gamma$-PGA]PHMeDOT $(\theta=7 \mathrm{~h})$ electrode. (b) Variation of the electrical conductivity with the strain for flexible [PEDOT/ $\gamma-\operatorname{PGA}] \operatorname{PHMeDOT}\left(\theta=\begin{array}{ll}7 & \text { h }\end{array}\right)$ electrodes. Error bars display standard deviations calculated considering five independent samples. (c) Flexible [PEDOT/ $\gamma$-PGA]PHMeDOT $(\theta=7 \mathrm{~h})$ electrode used power the LED bulb. (d) Energy harvesting system constructed using a Teflon holder with a stainless steel (AISI 304) disk and the $[\mathrm{PEDOT} / \gamma-\mathrm{PGA}] \mathrm{PHMeDOT}(\theta=7 \mathrm{~h})$ electrode. Schematic diagram of the circuits used to (e) charge and to (f) power the LED using the energy-harvesting device displayed in (d). (g) Photographs of the device used to power the LED bulb. 
Table 1. Properties of the studied systems: Swelling ratio (SR), voltammetric stored charge per surface unit $(Q)$, and the areal specific capacitance $(S C)$ as determined by cyclic voltammetry.

\begin{tabular}{cccc}
\hline System & SR $(\%)$ & $\boldsymbol{Q}\left(\mathbf{C} / \mathbf{c m}^{2}\right)$ & $S C\left(\mathbf{m F} / \mathbf{c m}^{2}\right)$ \\
\hline$\gamma$-PGA & 54 & $8.298 \cdot 10^{-3}$ & $2.4 \pm 0.3$ \\
PEDOT $/ \gamma$-PGA & 115 & $7.277 \cdot 10^{-3}$ & $2.6 \pm 0.4$ \\
{$[$ PEDOT $/ \gamma$-PGA]PHMeDOT $(\theta=6$ min $)$} & 206 & $7.981 \cdot 10^{-3}$ & $2.7 \pm 0.5$ \\
{$[$ PEDOT $/ \gamma$-PGA]PHMeDOT $(\theta=7 \mathrm{~h})$} & 289 & 0.1454 & $45.4 \pm 0.7$ \\
\hline
\end{tabular}


(a)

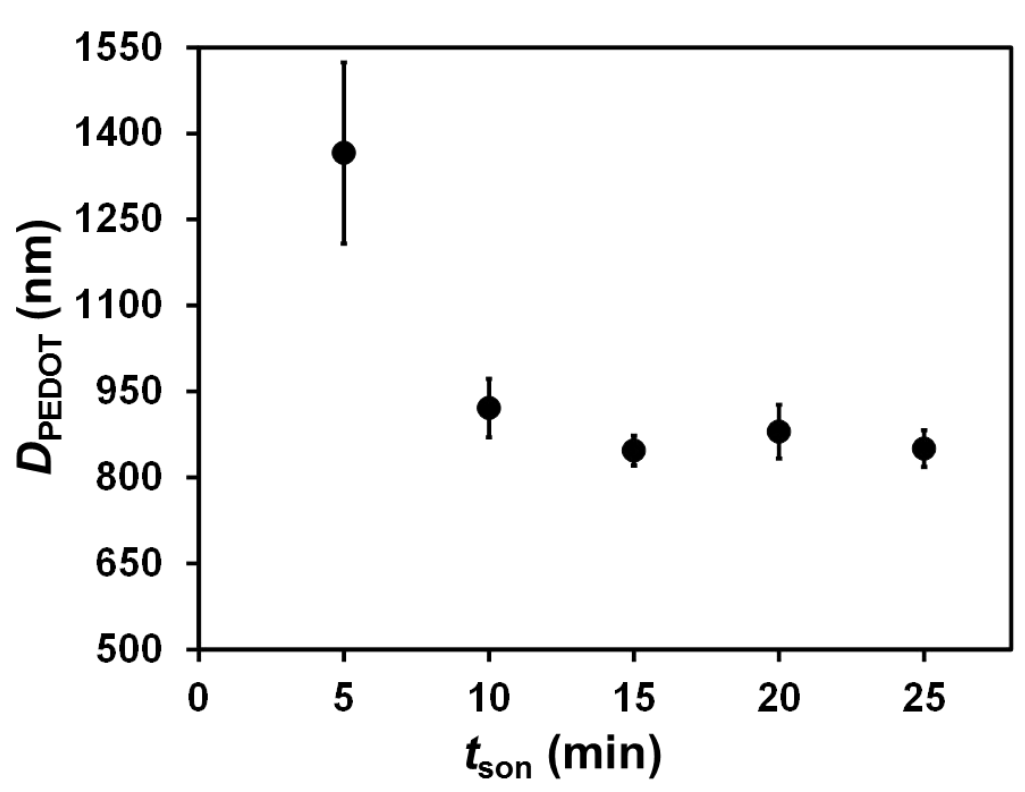

(b)

(c)

(d)

Figure 1 

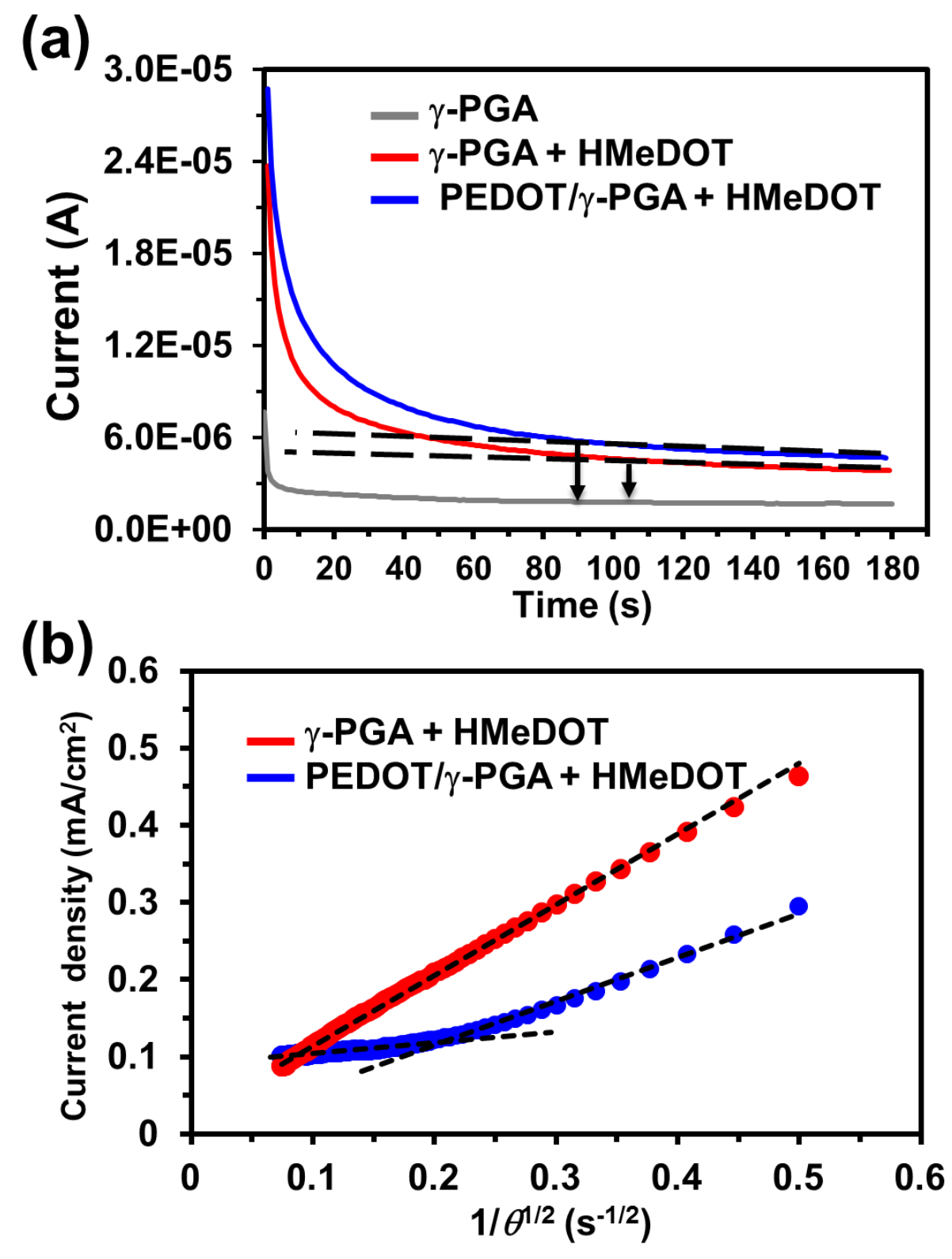

Figure 2 

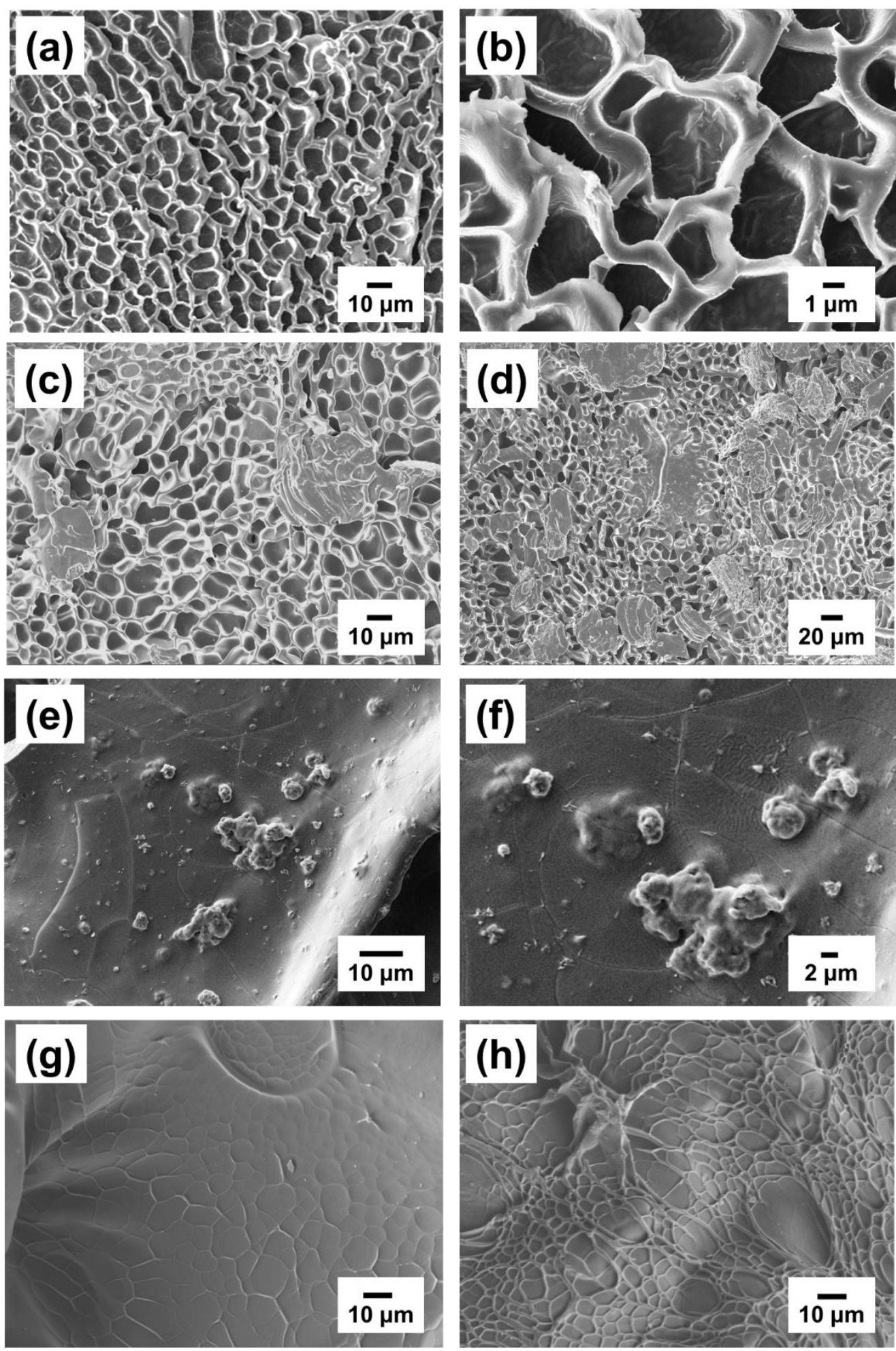

Figure 3 

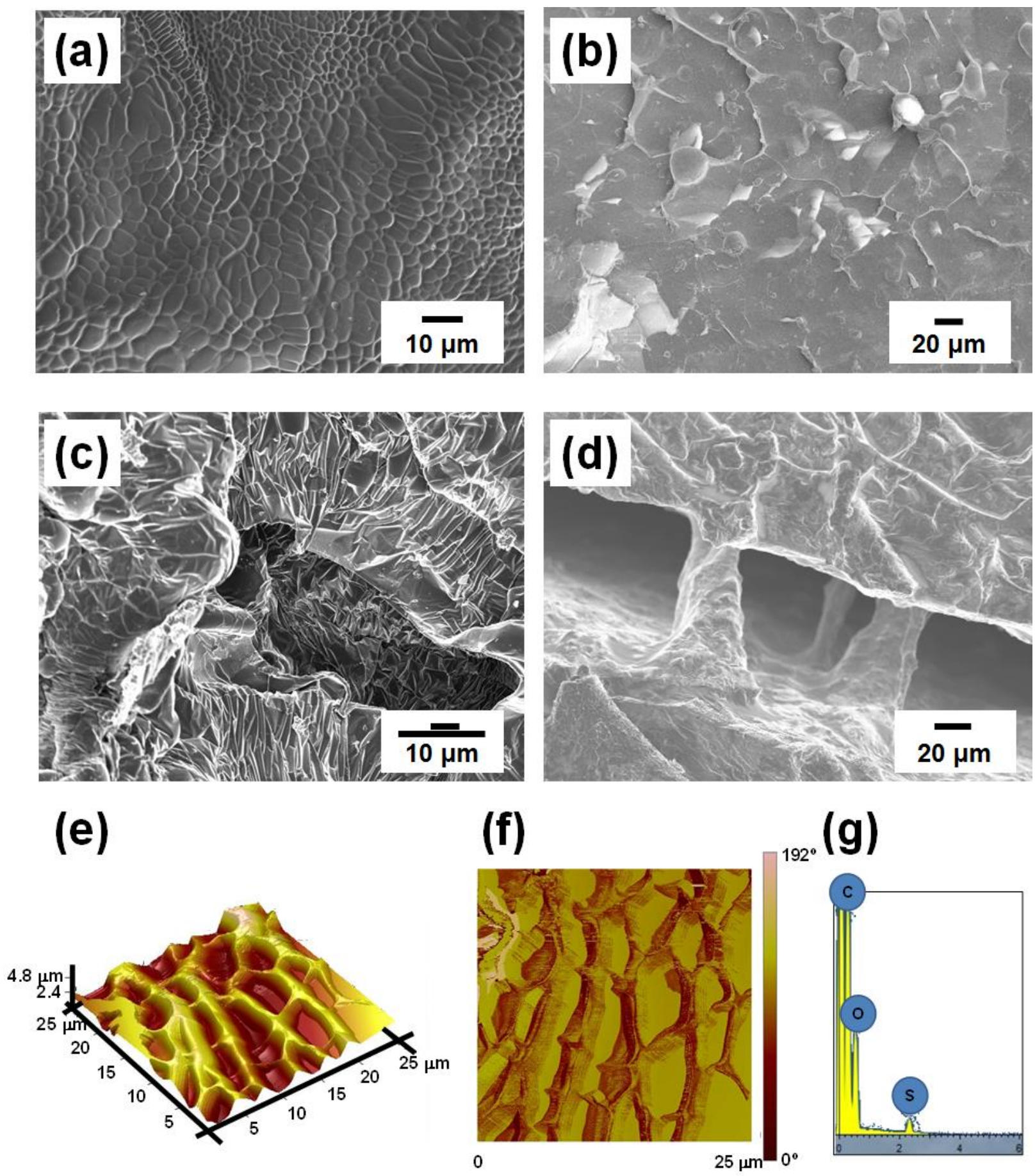

Figure 4 

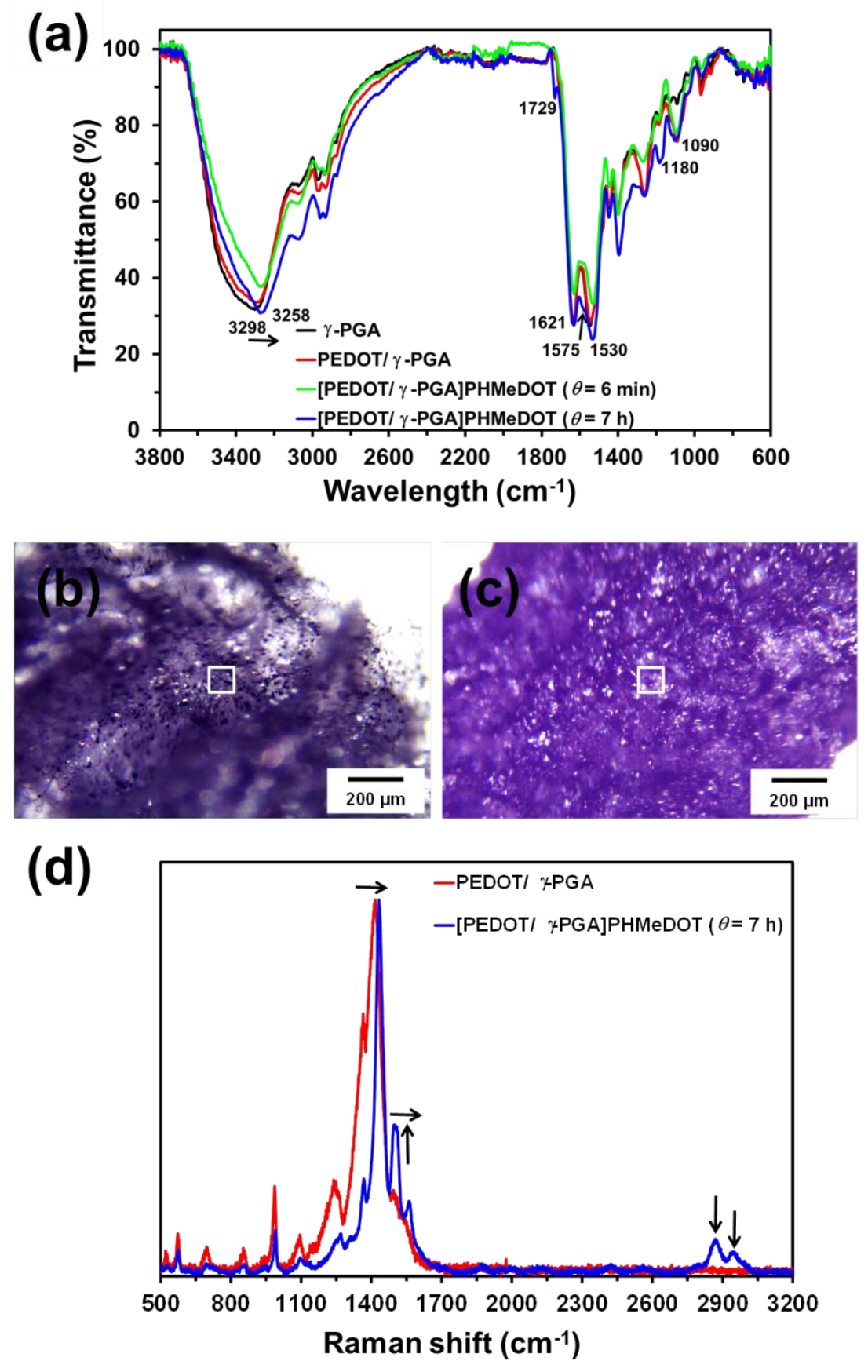

Figure 5 


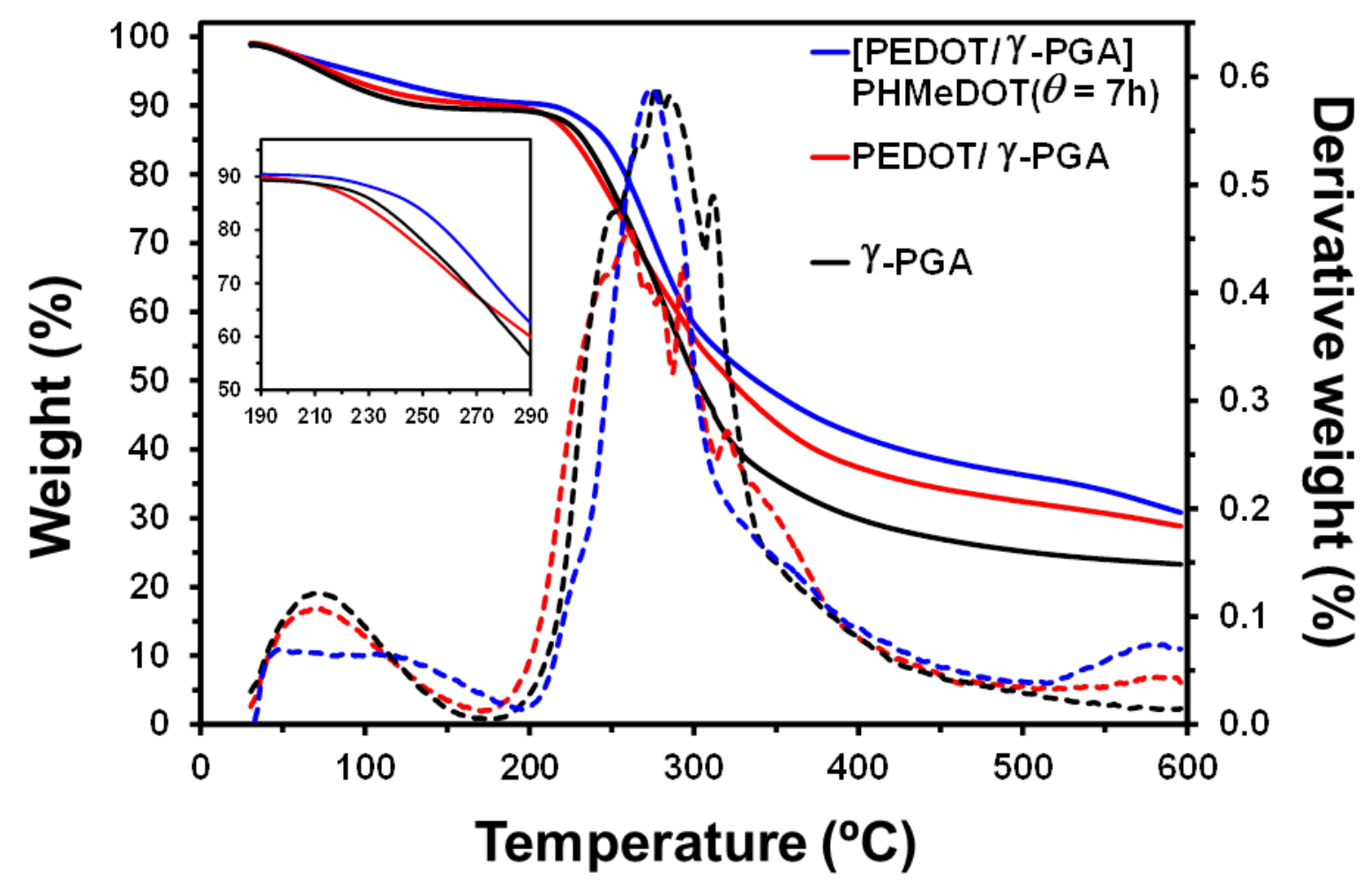

Figure 6 
(a)

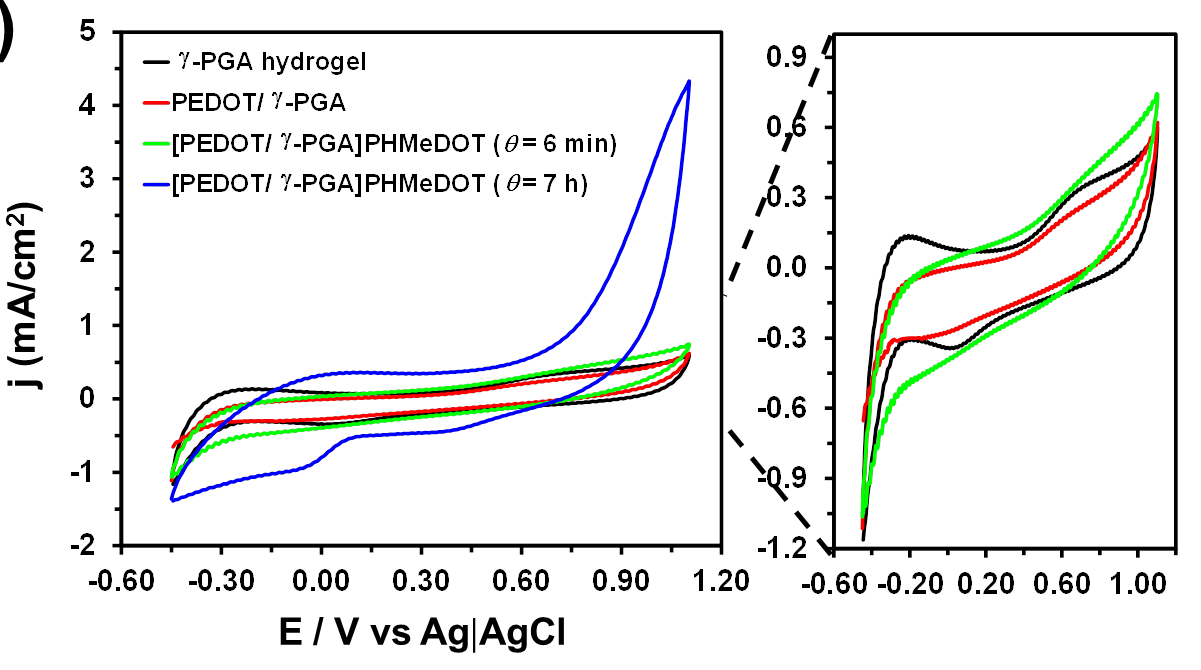

(b)

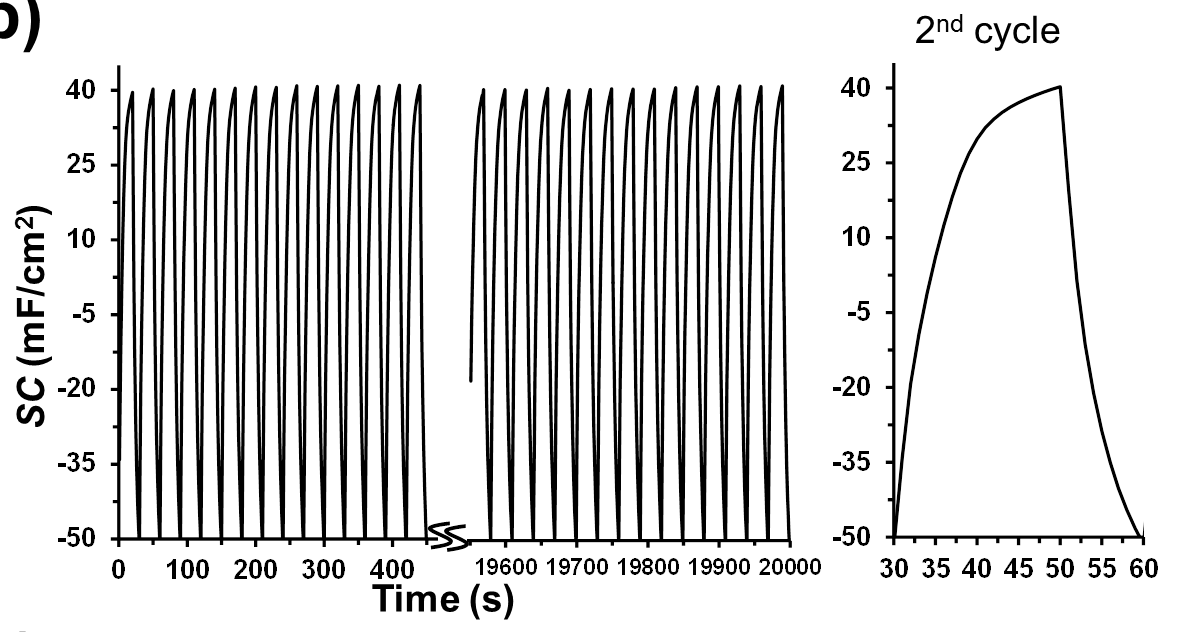

(c)
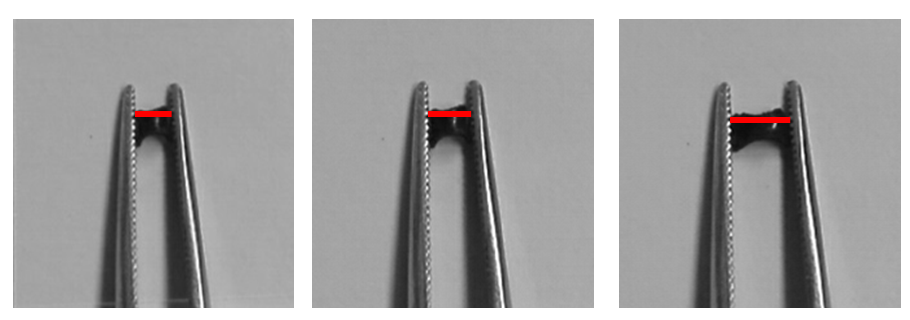

Figure 7 

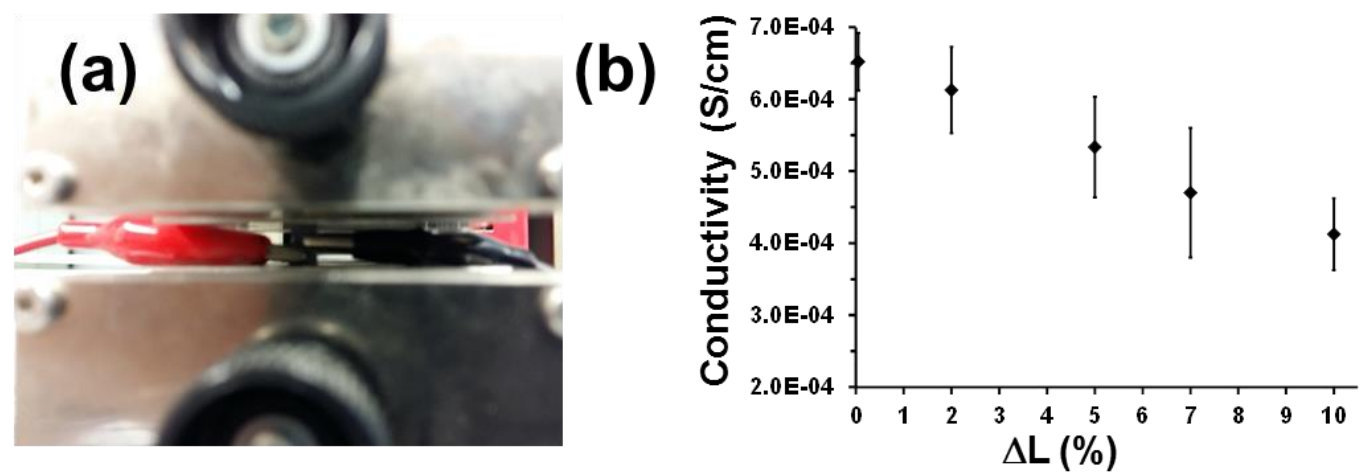

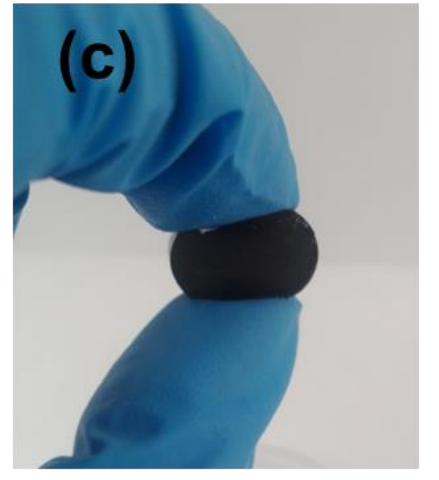

(e)

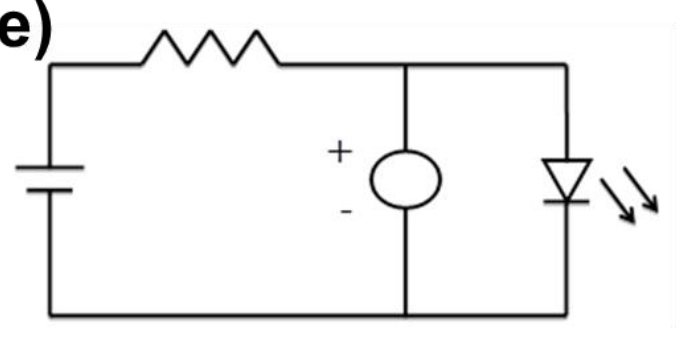

(f)
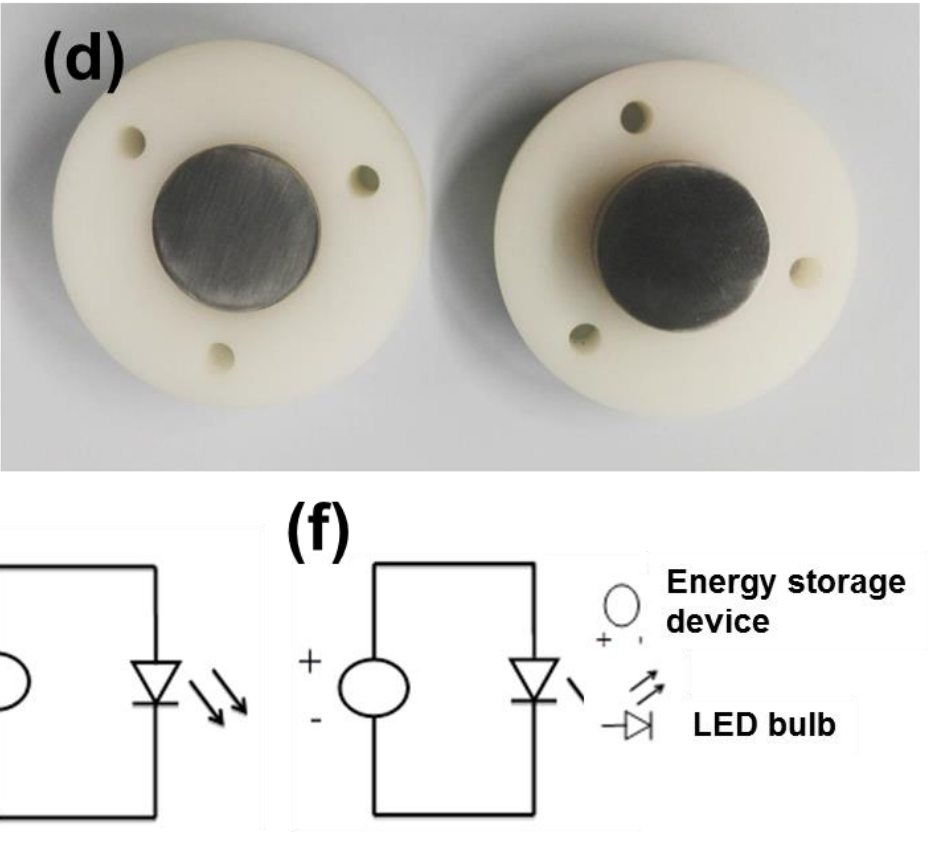

\section{(g)}

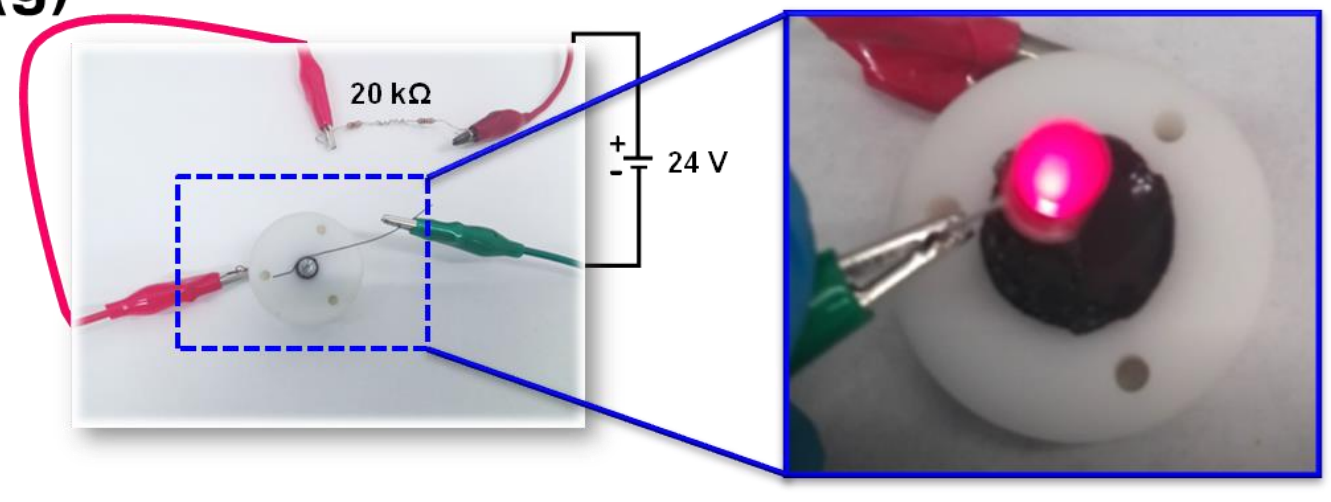

Figure 8 


\section{TOC graphic}

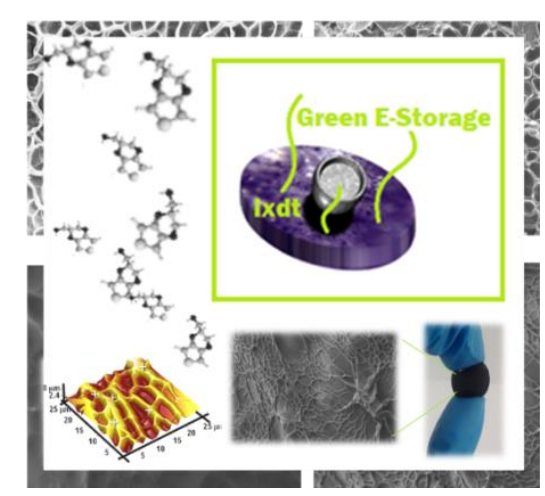

